Revue internationale P.M.E.

Économie et gestion de la petite et moyenne entreprise

Revure

internationale

PME

\title{
Le champ de l'entrepreneuriat : historique, évolution, tendances
}

\section{Louis Jacques Filion}

Volume 10, numéro 2, 1997

URI : https://id.erudit.org/iderudit/1009026ar

DOI : https://doi.org/10.7202/1009026ar

Aller au sommaire du numéro

Éditeur(s)

Presses de l’Université du Québec

ISSN

0776-5436 (imprimé)

1918-9699 (numérique)

Découvrir la revue

Citer cette note

Filion, L. (1997). Le champ de l'entrepreneuriat : historique, évolution, tendances. Revue internationale P.M.E., 10(2), 129-172.

https://doi.org/10.7202/1009026ar
Résumé de l'article

Ce texte présente une synthèse de la recherche sur l'entrepreneur et le propriétaire-dirigeant de PME. Dans un premier temps, les pionniers du domaine sont introduits : Cantillon, Say et Schumpeter. Les apports de plusieurs autres économistes dont Knight, Hayek, Penrose, Kirzner et Casson sont mentionnés. Dans un deuxième temps, les contributions des behavioristes, avec une attention particulière portée sur celle de McClelland, sont présentées. On y traite des caractéristiques les plus couramment attribuées aux entrepreneurs. À compter des années 1980, on observe un éclatement du champ de l'entrepreneurlat qui devient l'objet d'étude de presque toutes les disciplines des sciences humaines. Un certain nombre de typologies parmi les plus couramment étudiées en entrepreneuriat sont répertoriées ; une typologie de propriétaires-dirigeants de PME est aussi suggérée en annexe par l'auteur. En outre sont présentées un certain nombre de tendances théoriques, une définition de l'entrepreneur et, à la fin, quelques réflexions sur l'orientation du domaine. 


\title{
Le champ de l'entrepreneuriat: historique, évolution, tendances
}

\author{
Louis Jacques FILION \\ École des HEC de Montréal
}

"Je suis mal-aisé à esbranler; mais estant avoyé, je vais tant qu'on veut.

J'estrive autant aux petites entreprises qu'aux grandes... "

Michel de Montaigne, Essai

\section{MOTS CLÉS}

\section{Entrepreneuriat - Entrepreneurship - PME - Entrepreneurs Typologies entrepreneurs - Épistémologie entrepreneuriat Définition entrepreneur - Théorie entrepreneurs}

\begin{abstract}
RÉSUMÉ
Ce texte présente une synthèse de la recherche sur l'entrepreneur et le propriétaire-dirigeant de PME. Dans un premier temps, les pionniers du domaine sont introduits : Cantillon, Say et Schumpeter. Les apports de plusieurs autres économistes dont Knight, Hayek, Penrose, Kirzner et Casson sont mentionnés. Dans un deuxième temps, les contributions des behavioristes, avec une attention particulière portée sur celle de McClelland, sont présentées. On y traite des caractéristiques les plus couramment attribuées aux entrepreneurs.
\end{abstract}

\section{L'AUTEUR}

Louis Jacques Filion est professeur titulaire et titulaire de la Chaire d'entrepreneurship Maclean-Hunter à l'École des HEC de Montréal. II est spécialisé en entrepreneuriat et PME. II détient un B.A., un B.Sc. Soc., une M.A., une M.B.A. et un Ph.D. Cet auteur compte une expérience diversifiée de la pratique de la gestion: ressources humaines, gestion des opérations, marketing et direction générale. Conseiller en gestion, il a été rattaché au bureau de Montréal de Caron, Bélanger, Ernst et Young. Ses recherches portent sur les systèmes d'activités des entrepreneurs et des travailleurs autonomes ainsi que sur la stratégie des PME. II porte un intérêt particulier à la vision entrepreneuriale. Adresse: Chaire d'entrepreneurship Maclean-Hunter, École des Hautes Études commerciales, 3000, Chemin de la Côte Sainte-Catherine, Montréal (Québec), H3T 2 A7. 
À compter des années 1980 , on observe un éclatement du champ de l'entrepreneuriat qui devient l'objet d'étude de presque toutes les disciplines des sciences humaines. Un certain nombre de typologies parmi les plus couramment étudiées en entrepreneuriat sont répertoriées; une typologie de propriétaires-dirigeants de PME est aussi suggérée en annexe par l'auteur. En outre sont présentées un certain nombre de tendances théoriques, une définition de l'entrepreneur et, à la fin, quelques réflexions sur l'orientation du domaine.

\section{ABSTRACT}

This text presents a summary of the research on entrepreneurs and ownermanager. First, it introduces the pioneers of the field: Cantillon, Say and Schumpeter. The contributions of a number of other economists, including Knight, Hayek, Penrose, Kirzner and Casson are mentioned. It then describes the contributions of the behaviourists, with special emphasis on McClelland. The characteristics most often attributed to entrepreneurs are discussed. From the 1980s onwards, the field of entrepreneurship exploded and was appropriated by almost all the soft science disciplines. Some of the most commonly studied typologies in entrepreneurship are introduced, and a typology of small business owners-managers is proposed by the author in an appendix. A number of theoretical trends are discussed. A definition of the entrepreneur is suggested and the text also contains some reflections on future directions in the field.

\section{RESUMEN}

Este texto presenta una sintesis de la investigación sobre el emprendedor y el propietario - dirigente de la PyME. En primer lugar, se introducen los pioneros del campo de investigación: Cantillon, Say y Schumpeter. Se mencionan también las aportaciones de otros muchos economistas como Knight, Hayek, Penrose, Kirzner y Casson. En segundo lugar se presentan las contribuciones de los behavioristas, con un énfasis particular en la de McClelland. Se discuten las características más frecuentemente atribuidas a los emprendedores. $A$ partir de los años 80 , se observa una apertura en el campo del emprendimiento, del que se apropian casi todas las disciplinas de las ciencias humanas. Se comentan un cierto número de tipologías de entre las más comunmente estudiadas sobre emprendimiento. Así, se sugiere en anexo una tipologia de propietarios - dirigentes de PyME. Se discuten un cierto número de tendencias a teorizar. Finalmente, se sugiere una definición de emprendedor y se aportan algunas reflexiones para la orientación del ámbito de estudio. 


\section{Introduction}

Parler de PME revient à parler de propriétaires-dirigeants de PME. Et on ne peut parler de propriétaires-dirigeants de PME sans parler de l'entrepreneur. L'approche et la façon de le faire ont varié d'un pays à l'autre, d'un continent à l'autre. Par exemple, en Grande-Bretagne, de Bolton en 1971 jusqu'aux années 1980, on a beaucoup parlé de propriétaires-dirigeants (Stanworth et al., 1982 ; Stanworth et Gray, 1991), mais on y parle de plus en plus d'entrepreneurs (Burns et Dewhurst, 1989 ; Birley, 1989 ; Deakins, 1996). Aux États-Unis, on y aborde les deux domaines, mais les études sur les entrepreneurs constituent un champ de recherche privilégié (Kent, Sexton et Vesper, 1982 ; Sexton et Smilor, 1986 ; Sexton et Kasarda, 1992).

La recherche sur l'entrepreneuriat a donné lieu à plus de 1000 publications par an présentées dans quelque 50 colloques et 25 revues spécialisées. Ce champ de recherche comprend de très nombreuses spécialisations. Nommonsen quelques-unes : innovation et créativité ; création, démarrage et fermeture d'entreprises ; croissance d'entreprises ; travail autonome et micro-entreprises ; franchises ; études sur diverses dimensions de l'entrepreneur : comportements, systèmes d'activités, processus entrepreneurial, intrapreneurs et entrepreneuriat corporatif, technopreneurs, développement régional, entrepreneuriat ethnique, systèmes de soutien à l'entrepreneuriat et politiques gouvernementales, entrepreneuriat coopératif, éducation entrepreneuriale, entrepreneuriat féminin ; études sur la PME avec tout ce que cela implique d'approches fonctionnelles, telles que finance de PME, marketing de PME, gestion des opérations PME, gestion des ressources humaines de la $\mathrm{PME}$, systèmes d'information et $\mathrm{PME}$, stratégie de PME.

Voilà quelques-unes des multiples composantes du champ de l'entrepreneuriat ; évidemment, ce texte ne les couvre pas toutes. S'adressant au débutant autant qu'à l'expert, cet article vise à présenter l'essentiel des connaissances sur l'entrepreneur et le dirigeant de PME. Dans un premier temps, nous aborderons le monde de l'entrepreneur tel que vu par les pionniers du domaine, les économistes, puis par ceux, qui, dans une autre discipline, se sont beaucoup intéressés à l'entrepreneur: les behavioristes. Ensuite, nous présenterons quelques illustrations de l'éclatement du champ et de son intégration dans presque toutes les disciplines des sciences humaines et administratives. Dans un deuxième temps, nous examinerons quelques typologies de propriétairesdirigeants, puis nous en suggérerons une, dégagée d'une taxonomie. Elle est présentée en annexe. Des implications de cette typologie pour la stratégie de la PME sont proposées. Diverses tentatives de théoriser dans le champ, surtout au cours de la dernière décennie, seront rapportées et brièvement commentées. Avant de conclure, nous présenterons une définition de l'entrepreneur qui se veut représentative des courants traditionnels de recherche dans le champ. 


\section{Le monde de l'entrepreneur}

Les gens qui œuvrent dans le domaine de l'entrepreneuriat diront qu'il existe une confusion peu commune relativement à la définition de l'entrepreneur. Nous préférons plutôt parler de différences dans les façons de définir l'entrepreneur. Chacun tend à voir et à définir l'entrepreneur à partir des prémisses de sa discipline. Vue sous cet angle, la confusion n'est peut-être pas si grande que l'on pense, puisque des éléments communs tendent à apparaitre dans chaque discipline. Par exemple, les économistes ont beaucoup associé l'entrepreneur à l'innovation, alors que les behavioristes ont beaucoup écrit sur les caractéristiques créatives et intuitives attribuées à l'entrepreneur. Nous y reviendrons.

\subsection{Les économistes}

Il faut d'abord nuancer la croyance populaire qui attribue l'origine de l'entrepreneuriat à la seule science économique. Une lecture attentive des deux premiers auteurs généralement identifiés comme les pionniers du domaine, Cantillon (1755) et Say $(1803,1815,1816$ et 1839), nous fait découvrir des auteurs qui s'intéressaient tant à l'économie qu'aux entreprises, à leur création, à leur développement et à leur gestion. Cantillon était essentiellement un banquier qu'on qualifierait aujourd'hui de prêteur de capitaux de risque. Ses écrits nous révèlent un homme à la recherche d'occasions d'affaires, préoccupé par une gestion astucieuse et économe qui optimalise le rendement sur le capital investi.

Vérin (1982) nous a montré l'origine et l'évolution du terme «entrepreneur ». On peut constater que le terme a acquis sa signification actuelle au cours du XVII ${ }^{e}$ siècle. Même si le terme était utilisé avant Cantillon, on peut remarquer, comme Schumpeter $(1954$, p. 222) l'a noté, que Cantillon fut le premier à présenter une conception claire de l'ensemble de la fonction de l'entrepreneur.

Certains auteurs ont associé Cantillon à l'une ou l'autre école de pensée. Cela est peu plausible, car Cantillon était un individualiste. Bien que les circonstances de sa vie ne l'aient pas amené à prendre racines dans un pays en particulier, il a manifesté un intérêt pour les questions économiques et un besoin de rationalité caractéristiques de plus d'un de ses contemporains européens de l'époque. Il est peut-être utile de mentionner ici que la famille Cantillon était originaire de Normandie. Elle a émigré en Irlande à l'époque de Guillaume le Conquérant qui leur avait confié l'administration d'un petit territoire, l'équivalent d'un comté. Il s'agissait donc d'une famille de petite noblesse. Mais Richard, dont on ignore la date de naissance - à ne pas confondre avec son oncle, le chevalier Richard Cantillon qui a, lui aussi, vécu à Paris -, se réfugia à Paris en 1716 à la suite de la chute des Stuart en GrandeBretagne. À cette époque, on retrouvait à Paris une communauté importante d'immigrants d'origine irlandaise, et Richard Cantillon agissait en quelque 
sorte comme un des parrains de cette communauté. Il vivait de ses rentes et recherchait des occasions d'investissement. Il voyageait beaucoup. Ses descriptions de la culture du thé aux Indes sont remarquables. Il savait analyser une opération, voir où elle était profitable et comment elle pourrait le devenir davantage. On peut d'ailleurs déceler des éléments précurseurs du taylorisme chez Cantillon ${ }^{1}$. Au début du XVII ${ }^{\mathrm{e}}$ siècle, il a même investi dans des opérations de postes de traite le long du Mississippi ${ }^{2}$. Cantillon était reconnu comme étant très près de ses sous, pour ne pas dire pingre. L'hypothèse a été émise par des contemporains que son cuisinier aurait volontairement mis le feu à sa propriété à Londres, où il s'était établi, à la suite du refus obstiné de son patron d'augmenter ses émoluments. Cet incendie entraîna la mort de Cantillon en 1734. Son manuscrit a été publié à titre posthume plus de 20 ans après son décès, après avoir beaucoup circulé à Paris ainsi qu'à Londres, et après avoir été corrigé par l'éditeur.

Jean-Baptiste Say est le deuxième auteur qui s'est beaucoup intéressé aux activités de l'entrepreneur. Il voyait le développement de l'économie par la création d'entreprises. Il rêvait de voir la révolution industrielle anglaise se transposer en France (Say, 1816). On l'a qualifié d'économiste, car, à l'époque et jusqu'à la moitié du $\mathrm{xx}^{\mathrm{e}}$ siècle, les sciences de l'administration étaient inexistantes. On qualifiait alors d'économiste toute personne qui s'intéressait aux organisations, qui parlait de création et de distribution de richesses. Si on devait catégoriser Say de nos jours, il se rapprocherait sans doute davantage d'un Peter Drucker que d'un Kenneth Galbraith. On peut déjà observer, à partir des écrits de Cantillon et de Say, que leur intérêt pour l'entrepreneuriat ne facilite pas leur identification à une discipline donnée. Ce sera là le lot de presque tous ceux qui s'intéresseront à ce domaine : ils l'analysent à partir des prémisses d'une discipline, mais dès qu'ils s'avancent un peu, ils débordent les frontières de cette discipline, ne s'y retrouvent plus aussi bien et n'y sont plus toujours aussi bien reconnus.

Cantillon et Say voyaient l'entrepreneur surtout comme un preneur de risques puisqu'il investissait son propre argent. Pour Cantillon, l'entrepreneur achète une matière première - souvent un produit de l'agriculture - à un prix certain pour la transformer et la revendre à un prix incertain. C'est donc

1. De même d'ailleurs qu'Olivier de Serre, cent cinquante ans avant lui, comme on peut le voir dans son Théâtre d'agriculture et messages des champs, $3^{\mathrm{C}}$ édition, 1605.

2 Ce sont dans ces mêmes postes que la famille Perreault de Québec et de Trois-Rivières a exploité. Le père de François-Joseph Perreault ira d'ailleurs s'établir à Saint-Louis après la conquête de 1760. Rappelons que François-Joseph Perreault est considéré comme un des pères de l'éducation au Québec, pour y avoir créé les premières écoles privées au début du $\mathrm{XIX}^{\mathrm{e}}$ siècle. 
quelqu'un qui sait saisir une occasion en vue de réaliser un profit, mais qui doit en assumer les risques. Say fera une différence entre l'entrepreneur et le capitaliste, entre les profits de l'un et de l'autre (Say, 1803; 1827, p. $295 ; 1815$; 1816 , p. 28-29; Schumpeter, 1954, p. 555). En ce sens, il associe l'entrepreneur à l'innovation; il voit l'entrepreneur comme un agent de changement. Entrepreneur lui-même, il est le premier à avoir défini l'ensemble des paramètres de ce que fait l'entrepreneur dans le sens où nous l'entendons de nos jours. Schumpeter (1954) lui-même a observé qu'une grande partie de sa contribution a consisté à faire connaître aux Anglo-Saxons la compréhension du monde de l'entrepreneur à partir des écrits de Jean-Baptiste Say. Compte tenu qu'il fut le premier auteur à constituer les assises du champ, nous l'avons qualifié de père du champ de l'entrepreneuriat (Filion, 1988).

Il est peut-être intéressant de mentionner que Say a essentiellement intégré dans ses écrits deux grands courants de pensée de son époque : celui des physiocrates et celui de la révolution industrielle en Grande-Bretagne. Grand admirateur d'Adam Smith, dont il a introduit les idées en France, de même que de la révolution industrielle anglaise (Say, 1816), il essaiera d'établir un cadre de pensée pour qu'elle devienne possible en France. Il appliquera à l'entrepreneur la pensée libérale proposée par Quesnay, Mercier de La Rivière, Mirabeau, Condorcet, Turgot et autres physiocrates pour développer l'agriculture.

Mais c'est Schumpeter qui donne son envol au domaine de l'entrepreneuriat et il l'associe nettement à l'innovation.

L'essence de l'entrepreneuriat se situe dans la perception et l'exploitation de nouvelles opportunités dans le domaine de l'entreprise [...] cela a toujours à faire avec l'apport d'un usage différent de ressources nationales qui sont soustraites de leur utilisation naturelle et sujettes à de nouvelles combinaisons. (Schumpeter, 1928)

Non seulement Schumpeter associe-t-il l'entrepreneur à l'innovation, mais l'ensemble de son œuvre imposante fait ressortir l'importance du rôle joué par l'entrepreneur dans le développement économique.

En fait, il n'est pas le seul à associer l'entrepreneuriat à l'innovation. Clark (1899) l'avait clairement fait avant lui, Higgins (1959), Baumol (1968), Schloss (1968), Leibenstein (1978) ainsi que la plupart des économistes qui s'intéressent à l'entrepreneuriat après lui le feront aussi. Ce que recherchent les économistes, c'est avant tout de mieux comprendre le rôle que joue l'entrepreneur dans le système économique (Smith, 1776; Mill, 1848 ; Knight, 1921 ; Innis, 1930, 1956 ; Baumol, 1968 ; Broehl, 1978 ; Leff, 1978, 1979 ; Kent, Sexton et Vesper, 1982). En ce sens, les économistes voient l'entrepreneur autant comme un « déceleur» d'occasions d'affaires (Higgins, 1959; Penrose, 1959; Kirzner, 1976) et un créateur d'entreprises (Ely et Hess, 1893 ; Oxenfeldt, 1943 ; Schloss, 1968) qu' un preneur de risques (Leibenstein, 1968 ; Kihlstrom 
et Laffont, 1979 ; Buchanan et Di Pierro, 1980). Hayek (1937 ; 1959) a montré que le rôle de l'entrepreneur consistait à informer le marché des nouveautés qu'il y présente. Knight (1921) a montré que l'entrepreneur assume un risque à cause de l'état d'incertitude dans lequel il évolue et qu'il est rémunéré en conséquence par le profit qu'il tire de l'activité dont il est l'initiateur. Hoselitz $(1952,1968)$ a parlé d'un niveau de tolérance plus élevé à travailler dans des conditions d'ambiguïté et d'incertitude chez les entrepreneurs. Casson (1982) fait une tentative intéressante pour mettre au point une théorie où sont associés entrepreneurs et développement économique. Il y insiste sur la dimension de la coordination de ressources et de la prise de décision. Leibenstein (1979) avait déjà établi un modèle où il mesurait le degré d'efficacité et d'inefficacité dans l'utilisation de leurs ressources par les entrepreneurs.

On parle de l'entrepreneur en économie, mais on l'a peu ou pas intégré dans les modèles classiques de développement économique. Lorsqu'on l'a fait, l'entrepreneur est représenté par une fonction. Les économistes qui se sont intéressés à l'entrepreneur sont demeurés la plupart du temps, comme c'est le cas dans d'autres disciplines, des marginaux. Si on voulait faire une synthèse des principaux courants de pensée en économie concernant l'entrepreneuriat, on serait amené à accepter la suggestion de Baumol (1993) et de faire référence à deux catégories d'entrepreneur : l'entrepreneur-organisateur d'entreprises et l'entrepreneur-innovateur. Le premier représente l'entrepreneur classique décrit par Say (1803), Knight (1921) et Kirzner (1983), tandis que le second représente celui décrit par Schumpeter (1934).

Il n'est jamais facile d'introduire des éléments de rationalité dans le comportement complexe de l'entrepreneur. Une des critiques qu'on peut formuler à l'égard des économistes, c'est de n'avoir pas su faire évoluer la science économique et d'avoir été incapable de créer une science du comportement économique de l'entrepreneur. Casson (1982) s'est rendu aux limites de ce qui est quantifiable et acceptable à la science économique. Le refus par les économistes d'accepter des modèles que l'on pourrait qualifier de non quantifiables marque les limites de cette science en entrepreneuriat. Cela amènera le monde de l'entrepreneuriat à se tourner vers les behavioristes pour mieux comprendre le comportement de l'entrepreneur.

\subsection{Les behavioristes}

Nous avons inclus sous le vocable «behavioristes », les psychologues, psychanalystes, sociologues et autres spécialistes du comportement humain. Un des premiers de cette discipline à s'intéresser aux entrepreneurs fut Max Weber (1930). Il a identifié le système de valeur comme fondamental pour expliquer le comportement des entrepreneurs. Il les voyait comme des innovateurs, des gens 
indépendants possédant une sorte d'autorité formelle par leur rôle de dirigeants d'entreprise. Mais celui qui a donné le coup d'envoi aux sciences du comportement au regard des entrepreneurs fut sans doute David C. McClelland.

\subsubsection{McClelland}

Au cours des années 1950, on s'interroge sur la montée de l'URSS : on se demande si l'homosoviéticus ne va pas supplanter l'homo americanus. C'est ce qui amène David $\mathrm{C}$. McClelland à se pencher sur l'histoire afin d'expliquer le pourquoi des grandes civilisations. À partir de cette étude remarquable, McClelland (1961) identifie plusieurs éléments, mais essentiellement la présence de héros dans la littérature. Les générations suivantes prennent ces héros comme modèles et ont tendance à les imiter dans leurs comportements. Ces héros ont tendance à faire sauter les barrières, à repousser la limite de ce qu'il est possible d'accomplir. Selon McClelland, les gens formés sous cette influence développent un fort besoin de réalisation, et il associe ce besoin à l'entrepreneur. Toutefois, il ne définit pas l'entrepreneur de la même façon qu'on le fait en général dans la littérature du domaine. Voici sa définition:

Un entrepreneur est quelqu'un qui exerce un contrôle sur une production qui ne sert pas qu'à sa consommation personnelle. Suivant ma définition, par exemple, un gestionnaire dans une unité de production d'acier en U.R.S.S. est un entrepreneur. (McClelland, 1971 ; voir aussi 1961, p. 65)

En fait, McClelland (1971) a surtout étudié des gestionnaires de grandes organisations. Bien qu'ils soient fortement associés au domaine de l'entrepreneuriat, ses écrits ne font ressortir aucune relation entre le besoin de réalisation et le fait de lancer, de posséder, ou même de gérer une entreprise (Brockhaus, 1982, p. 41). Mentionnons que McClelland a aussi identifié le besoin de puissance, mais c'est là une dimension de sa recherche à laquelle il a peu donné suite et dont on parle moins. Plusieurs chercheurs ont étudié le besoin de réalisation, mais personne ne semble avoir atteint des résultats vraiment concluants en ce qui a trait à l'association de ce concept à la réussite de l'entrepreneur (Durand et Shea, 1974 ; Hundall, 1971 ; Schrage, 1965; Singh, 1970; Singh et Singh, 1972).

Alors que certains ont trouvé que le besoin de réalisation était insuffisant pour expliquer la création d'entreprises (Hull, Bosley et Udeel, 1980), d'autres ont considéré la seule formation au besoin de réalisation comme insuffisante pour expliquer le succès de l'entrepreneur (Durand, 1975 ; Neck, 1971, Patel, 1975 ; Timmons, 1971). Timmons (1973) a cependant trouvé que ceux qui avaient suivi des sessions de formation pour augmenter leur besoin de réalisation avaient créé des entreprises dans une proportion plus grande que les 
autres groupes. Gasse (1978) a observé que McClelland a restreint ses recherches aux secteurs d'activité économique. Cette observation nous apparaît fort pertinente, car le besoin de réalisation s'exprimera en fonction des valeurs prédominantes d'une société donnée. Dans l'ex-URSS, cela aurait pu signifier de devenir un membre influent du parti communiste; dans l'Europe féodale, d'accéder à la noblesse; dans le Québec du XIX ${ }^{\mathrm{e}}$ siècle, de jouer un rôle influent dans la hiérarchie des institutions catholiques. Ainsi, pour que le besoin de réalisation soit vraiment canalisé vers l'entrepreneuriat et la création d'entreprises, il faut que les valeurs sociales ambiantes reconnaissent et valorisent suffisamment la réussite en affaires pour y attirer celles et ceux qui ont un fort besoin de réalisation. À la suite de Gunder (1969) et de Kunkel (1965), Gasse (1982) observe avec justesse que la théorie du besoin de réalisation de McClelland n'est pas satisfaisante, car elle ne peut considérer les structures sociales déterminantes sur les orientations individuelles. En somme, il est difficile d'expliquer le choix de créer une entreprise ou de la réussite d'un entrepreneur à partir de son besoin de réalisation (Brockhaus, 1982).

Une deuxième critique à formuler à l'égard de cette théorie du besoin de réalisation de McClelland porte sur sa simplicité. McClelland a tenté d'expliquer le développement et la prospérité d'une société par seulement deux facteurs déterminants: le besoin de réalisation et le besoin de puissance. Comme c'est le cas pour les personnes et les organisations, il est douteux que l'on puisse expliquer le comportement des sociétés par seulement un ou deux facteurs. Marx $(1844,1848)$ a insisté sur le rôle des idéologies, Weber (1930) et Tawney (1947) sur celui des idéologies religieuses, Burdeau (1979) et Vachet (1988) sur celui de l'idéologie libérale, mais Kennedy (1991), Rosenberg et Birdzell (1986), ainsi que Toynbee (1994) ont bien montré la multiplicité de facteurs qui expliquent le développement des sociétés et des civilisations.

Brockhaus (1982) a souligné que le lien entre la propriété d'une petite entreprise et un besoin de réalisation élevé est loin d'avoir été prouvé. Toutefois, McClelland aura certainement démontré que l'être humain est un produit social. On peut penser que les hommes tendent à reproduire leur propre modèle. En ce sens, on sait que le rôle de modèle explique dans un grand nombre de cas la décision de lancer une entreprise (Filion, 1988, 1990a, 1991a et b). On peut ainsi penser que, toutes choses étant égales par ailleurs, plus le nombre d'entrepreneurs dans une société sera élevé, plus nombreux et valorisés seront les modèles d'entrepreneurs présents, plus nombreux seront les jeunes qui tendront à imiter ces modèles, c'est-à-dire à choisir l'entrepreneuriat comme voie de carrière. 


\subsubsection{L'école des traits de caractère}

Après McClelland, les behavioristes vont dominer la discipline de l'entrepreneuriat pendant 20 ans, jusqu'au début des années 1980 . On cherche à savoir qui est l'entrepreneur, quelles sont ses caractéristiques. Les sciences du comportement sont en pleine expansion. On a établi de meilleurs consensus que dans d'autres disciplines quant aux méthodologies de recherche qui offrent validité et fiabilité. Ce mouvement se reflète par des recherches sur plusieurs sujets et, en particulier, sur les entrepreneurs. Des milliers de recherches montreront toute une série de caractéristiques attribuées aux entrepreneurs. Le tableau 1 ci-dessous présente les plus courantes.

\section{TABlEaU 1}

Caractéristiques le plus souvent attribuées aux entrepreneurs par les spécialistes en comportement

\begin{tabular}{ll}
\hline Innovateurs & Besoin de réalisation \\
Leaders & Internalité \\
Preneurs de risques modérés & Confiance en soi \\
Indépendants & Implication à long terme \\
Créateurs & Tolérance à l'ambiguité et à l'incertitude \\
Énergiques & Initiative \\
Persévérants & Apprentissage \\
Originaux & Utilisation de ressources \\
Optimistes & Sensibilité envers les autres \\
Orientés vers les résultats & Agressivité \\
Flexibles & Tendance à faire confiance \\
Débrouillards & Argent comme mesure de performance \\
\hline
\end{tabular}

Sources: Hornaday, 1982, «Research about living entrepreneurs », dans C.A. Kent et D.L. Sexton et al. (éds), Encyclopedia of Entrepreneurship, Englewood Cliffs, N.J., Prentice-Hall, p. 20-34.

Meredith, Nelson et Neck, 1982, The Practice of Entrepreneurship, Genève, Bureau international du travail.

Timmons, 1978, "Characteristics and role demands of entrepreneurship », American Journal of Small Business, vol. 3, $\mathrm{n}^{\circ}$ 1, p. 5-17.

Ces nombreuses recherches fondées sur des méthodologies pourtant impeccables ont cependant produit des résultats fort variables et souvent contradictoires. À ce jour, on n'a pas encore établi un profil psychologique scientifique absolu de l'entrepreneur. Plusieurs raisons peuvent expliquer ce phénomène. D'abord, les différences dans les échantillonnages. On peut penser que la fonction crée l'organe et qu'une caractéristique se développe à l'exercice d'un métier. Ainsi, on peut penser qu'un échantillon d'entrepreneurs qui ont lancé une 
entreprise il y a deux ans ne conduira pas au même profil qu'un échantillon étudiant des entrepreneurs qui ont lancé une entreprise il y a 20 ans. Les formations et emplois antérieurs auront aussi une influence, ainsi que la religion, les valeurs du milieu d'éducation, la culture familiale, etc. Il faut aussi compter sur le fait que chaque chercheur propose sa définition de l'entrepreneur, qui varie bien sûr de l'un à l'autre. Pour certains est entrepreneur toute personne qui crée une entreprise, tandis que, pour d'autres, les entrepreneurs sont ceux qui ont créé des entreprises qui ont pris de l'expansion. Pour d'autres encore de l'école schumpétérienne est entrepreneur toute personne qui apporte des innovations. En analysant certains de ces résultats de recherche, on réalise qu'un même échantillonnage peut inclure des dirigeants de PME, des franchisés et des travailleurs autonomes.

Nous ne sommes pas arrivés au point où nous pourrions évaluer une personne et établir avec certitude si elle réussira ou non comme entrepreneur. On peut toutefois dire si cette personne possède des caractéristiques et des aptitudes qu'on retrouve le plus souvent chez les entrepreneurs. Même si l'on n'est pas arrivé à établir un profil scientifique absolu de ce qu'est l'entrepreneur, les recherches effectuées sur le sujet offrent des repères non négligeables à celles et ceux qui veulent devenir entrepreneur pour mieux se situer comme entrepreneurs potentiels. Les recherches sur les entrepreneurs à succès (Filion, 1991a et b) permettent aux entrepreneurs en exercice et en devenir de relever les caractéristiques sur lesquelles ils devront travailler pour mieux réussir.

Lorrain et Dussault (1988a) ont montré que les comportements sont de meilleurs prédicteurs de succès que les traits de personnalité. Après avoir connu son heure de gloire, l'école de pensée des traits de personnalité tend maintenant à disparaître. Kets de Vries (1985) avance simplement que les entrepreneurs sont des gens mal adaptés qui ont besoin de créer leur propre environnement. De nombreux chercheurs ont trouvé un cheminement identique et sont d'avis que les entrepreneurs créent des entreprises non pas tant par attirance pour le travail à leur compte qu'en réaction à un milieu de travail qui ne leur convient pas (Bannock, 1981 ; Chell, 1985 ; Collins, Moore et Unwalla, 1964 ; Collins et Moore, 1970 ; Du Toit, 1980; Scase et Goffee, 1980 ; Stanworth et Curran, 1973). D'autres chercheurs ont observé un degré de névrose plus élevé chez l'entrepreneur que parmi la population en général (Eysenck, 1967; Lynn, 1969). Cela peut se comprendre, car la nature des activités de l'entrepreneur l'amène à des états de déséquilibres personnels constants. On a d'ailleurs souvent remarqué qu'il faut une grande stabilité émotive pour bien «fonctionner» comme entrepreneur.

En réalité, une des conclusions à formuler en ce qui a trait aux caractéristiques des entrepreneurs peut se résumer dans l'homme social. L'être humain est un produit de son milieu. Plusieurs auteurs ont montré que les entrepreneurs 
reflètent les caractéristiques du temps et du lieu où ils ont évolué (Ellis, 1983; Gibb et Ritchie, 1981 ; McGuire, 1964, 1976 ; Newman, 1981 ; Toulouse, 1979). Considéré dans l'optique du comportement de l'entrepreneur, l'entrepreneuriat apparaît d'abord comme un phénomène régional ; les cultures, les besoins, les habitudes d'une région façonnent des comportements. Les entrepreneurs les intègrent, les assimilent, les interprètent, et cela se reflète dans leur façon d'agir, de construire leur entreprise. À l'ère des médias électroniques, il est évident que les grands entrepreneurs exercent une influence qui dépasse les frontières de leur région et de leur pays. Il n'en demeure pas moins que le plus fort taux de fréquence de relations que la population entretient avec des entrepreneurs s'établit avec des entrepreneurs locaux. Et eux reflètent assez bien, généralement, la culture du milieu d'où ils sont issus. Ils constituent souvent de bonnes courroies de transmission des cultures régionales qu'ils font souvent eux-mêmes évoluer. On retrouve aussi ce phénomène en entrepreneuriat ethnique (Toulouse et Brenner, 1992).

De nombreuses recherches ont montré que vous avez plus de chances de devenir entrepreneur si vous avez un modèle dans votre famille ou dans votre environnement (Filion, 1988, 1991 a et b). Lorsque vous devenez entrepreneur, la nature de l'activité vous amène à pratiquer et à développer certaines caractéristiques. Par exemple, vous devez être tenace et créatif si vous voulez rester en affaires. Ainsi, en plus de l'être social, l'être qui apprend en exerçant un métier joue un rôle sur les caractéristiques de l'entrepreneur. Par exemple, on peut voir l'entrepreneur comme quelqu'un qui définit des projets et repère ce qu'il va devoir apprendre pour pouvoir les réaliser. À partir de cette facette, on peut voir l'entrepreneur tant comme quelqu'un qui doit continuer à apprendre pour s'adapter à un métier dont la nature des activités l'amène à demeurer en constante évolution, que comme quelqu'un qui définit des besoins d'apprentissage. L'entrepreneur doit non seulement définir ce qu'il doit faire, mais aussi ce qu'il doit apprendre pour être en mesure de le faire (Filion, 1988). Par exemple, Rotter (1966) a toujours considéré l'internalité comme une caractéristique acquise. Cela se comprend. Lorsque vous êtes dans une position de leadership, vous devez exercer une certaine influence sur les gens si vous voulez que les choses arrivent où vous le souhaitez. C'est là une des dimensions qui fait que votre leadership existe et se développe. Ainsi, l'internalité, caractéristique souvent attribuée aux entrepreneurs, est d'abord une compétence graduellement apprise et acquise par quelqu'un qui doit faire en sorte que ses desseins se réalisent. On sait que les entrepreneurs qui réussissent ont en général un niveau élevé d'internalité. Il ne s'agit cependant pas d'une caractéristique propre aux entrepreneurs, car on la retrouve dans plusieurs autres catégories de leaders et de gens qui réussissent. Des chercheurs ont aussi observé que l'entrepreneur a un fort besoin de contrôler son environnement, ce qui l'entoure (Filion, 1991a et b; Kets de Vries, 1985). 
En conclusion sur les recherches reliées au comportement de l'entrepreneur, on peut dire que nous ne sommes pas arrivés à établir un profil scientifique qui permettrait d'identifier à coup sûr un entrepreneur potentiel. Mais nous connaissons suffisamment de caractéristiques entrepreneuriales pour permettre à celles et à ceux qui veulent devenir entrepreneurs de pouvoir se situer par rapport à l'exercice de ce métier. On voit cependant que l'étude du comportement de l'entrepreneur n'est plus l'apanage des behavioristes. En effet, on observe de plus en plus que les recherches se déplacent vers d'autres sphères, telles que celle des compétences requises pour bien fonctionner comme entrepreneur, de même que vers les modes d'apprentissage personnels et organisationnels requis pour bien s'adapter à l'évolution des activités reliées à l'exercice du métier d'entrepreneur.

\section{L'éclatement de l'entrepreneuriat}

Les années 1980 allaient voir apparaître l'éclatement du domaine de l'entrepreneuriat vers la quasi-totalité des disciplines des sciences humaines et administratives. Deux événements allaient marquer cette transition : la publication d'une première encyclopédie sur l'état des connaissances dans le domaine (Kent, Sexton et Vesper, 1982), ainsi que la tenue d'un premier grand colloque annuel réservé à la recherche dans cette nouvelle discipline, soit celui de Babson.

En fait, la lecture de la table des matières des Actes de colloques annuels tel que celui de Babson intitulé «Frontiers of Entrepreneurship Research », ainsi que ceux du CIPE (Conseil international de la petite entreprise) nous permet de dégager les thèmes les plus fréquemment discutés lors de ces événements.

Nous avons retenu 25 thèmes dominants dans la discipline. Dans une des bibliographies la plus complète publiée sur le sujet, Harold P. Welsch (1992) en a retenu 27.

Il est intéressant d'observer que le développement de la discipline de l'entrepreneuriat diffère de celui des autres disciplines. En effet, on observe qu'une multitude de chercheurs, chacun à partir de la culture, de la logique, des méthodologies plus ou moins établies de sa propre discipline, s'intéressent et réalisent des recherches sur l'entrepreneuriat et les PME. On a vu apparaître les premiers détenteurs de doctorat dans le domaine au cours de la décennie 1980. Mais la grande majorité des gens qui s'intéressent au domaine viennent de disciplines autres que l'entrepreneuriat et ne font pas de l'entrepreneuriat leur champ principal d'activités. Leurs activités de recherche et d'enseignement en entrepreneuriat sont, le plus souvent, connexes à leurs activités principales. On observe qu'un nombre croissant de gens consacrent de plus en plus de temps et d'intérêt à l'entrepreneuriat. En réalité, on compte de plus en plus de créations d'entreprises, et la part du PIB occupée par les PME dans tous les 


\section{TABleAu 2}

\section{Thèmes principaux de recherche en entrepreneuriat}

- Caractéristiques comportementales des entrepreneurs

- Caractéristiques économiques et démographiques des PME

- Entrepreneuriat et PME dans les pays en développement

- Caractéristiques managériales des entrepreneurs

- Processus entrepreneurial

- Création d'entreprises

- Développement d'entreprises

- Capitaux de risque et financement de la PME

- Gestion des entreprises, redressements, acquisitions

- Entreprises de haute technologie

- Stratégie et croissance de l'entreprise entrepreneuriale

- Alliance stratégique

- Entrepreneuriat corporatif ou intrapreneuriat

- Entreprises familiales

- Travail autonome

- Incubateurs et systèmes de soutien à l'entrepreneuriat

- Réseaux

- Facteurs influençant la création et le développement d'entreprises

- Politique gouvernementales et création d'entreprises

- Femmes, groupes minoritaires, ethnicité et entrepreneuriat

- Éducation entrepreneuriale

- Recherche en entrepreneuriat

- Études culturelles comparatives

- Entrepreneuriat et société

- Franchises

pays augmente chaque année. Pour suivre l'évolution de même que les besoins de leurs étudiants ainsi que de leurs clients, bon nombre de professeurs se doivent de mieux connaître l'entrepreneuriat et la PME. Ainsi, l'appropriation et l'intégration de la discipline de l'entrepreneuriat dans les autres disciplines, surtout des sciences humaines et administratives, constituent un phénomène unique qui ne s'est jamais produit avec autant d'ampleur dans la construction paradigmatique d'aucune autre discipline dans les sciences humaines. Le tableau suivant (tableau 3) montre les grands blocs de recherche en entrepreneuriat.

On entend souvent dire qu'il existe une grande confusion dans le domaine de l'entrepreneuriat parce qu'il n'y a pas de consensus quant à la définition de l'entrepreneur et quant aux paramètres qui constituent le paradigme. On peut aussi présenter le point de vue inverse et dire que l'entrepreneuriat demeure un des rares sujets qui attire des spécialistes d'un aussi grand nombre de disciplines qui sont amenés à échanger, à examiner ce que font les autres dans les 
TABleau 3

Recherche en entrepreneuriat

\begin{tabular}{|c|c|c|c|}
\hline Clients & Sujets & Spécialistes & Méthodologies \\
\hline $\begin{array}{l}\text { Système } \\
\text { politique }\end{array}$ & $\begin{array}{l}\text { Politiques } \\
\text { gouvernementales } \\
\text { Développement } \\
\text { régional }\end{array}$ & $\begin{array}{l}\text { Économistes } \\
\text { Sociologues }\end{array}$ & Quantitatives \\
\hline $\begin{array}{c}\text { Entrepreneurs } \\
\text { Entrepreneurs } \\
\text { potentiels } \\
\text { Éducateurs }\end{array}$ & $\begin{array}{l}\text { Caractéristiques } \\
\text { de l'entrepreneur } \\
\text { Milieu } \\
\text { entrepreneurial }\end{array}$ & $\begin{array}{l}\text { Sciences } \\
\quad \text { du comportement } \\
\text { Sociologues } \\
\text { Anthropologues }\end{array}$ & $\begin{array}{l}\text { Quantitatives } \\
\text { et qualitatives }\end{array}$ \\
\hline $\begin{array}{l}\text { Entrepreneurs } \\
\text { Entrepreneurs } \\
\quad \text { potentiels } \\
\text { Éducateurs } \\
\text { Conseil aux } \\
\quad \text { entrepreneurs }\end{array}$ & $\begin{array}{l}\text { Pratiques } \\
\quad \text { des affaires } \\
\text { Activités de gestion } \\
\text { Financement } \\
\text { Leadership } \\
\text { Pensée stratégique }\end{array}$ & $\begin{array}{l}\text { Sciences } \\
\text { de la gestion }\end{array}$ & $\begin{array}{l}\text { Quantitatives } \\
\text { Qualitatives }\end{array}$ \\
\hline
\end{tabular}

disciplines connexes et à s'interroger sur la façon dont ils le font. En réalité, la confusion semble grande lorsqu'on compare les définitions de l'entrepreneur entre disciplines (Filion, 1987 et 1988). Mais on retrouve un consensus étonnamment large en ce qui a trait aux définitions et aux caractéristiques attribuées aux entrepreneurs par les spécialistes d'une même discipline. Chez les économistes, on s'entend pour associer l'entrepreneur à l'innovation; on le voit comme une dynamo du développement. Chez les spécialistes des sciences du comportement, on attribue à l'entrepreneur des caractéristiques de créativité, de ténacité, d'internalité et de leadership. Chez les ingénieurs et les spécialistes de la gestion des opérations, l'entrepreneur est perçu comme un bon répartiteur et un coordonnateur de ressources. Chez les spécialistes de la finance, l'entrepreneur sait mesurer le risque. Pour les spécialistes de la gestion, l'entrepreneur sait se donner des fils conducteurs, des visions autour desquelles il organise l'ensemble de ses activités; il sait se débrouiller et s'organiser. Il excelle dans l'organisation et l'utilisation des ressources qui l'entourent. Pour les spécialistes du marketing, l'entrepreneur identifie des occasions d'affaires, sait «faire autrement » et penser «clients». Pour celles et ceux qui étudient la création d'entreprises, les meilleurs éléments pour prédire le succès futur de l'entrepreneur demeurent la valeur, la diversité ainsi que la profondeur de l'expérience de même que des expertises acquises par l'entrepreneur potentiel du secteur dans lequel il veut se lancer. 
En somme, le domaine de l'entrepreneuriat a intéressé les spécialistes de presque toutes les disciplines des sciences humaines au cours de la dernière décennie. La confusion qui semble y exister reflète essentiellement les logiques et les cultures propres à chacune de ces disciplines. On peut penser que l'entrepreneuriat pourra devenir au cours de la prochaine décennie un des axes de ralliement de l'ensemble des sciences humaines, car c'est un des rares sujets qui a su attirer un nombre aussi considérable de spécialistes de disciplines aussi diverses.

\section{Typologies d'entrepreneurs, de PME et de propriétaires dirigeants de PME}

On ne peut traiter du domaine de l'entrepreneuriat sans parler de typologies d'entrepreneurs. L'intérêt pour les typologies est aussi grand chez les chercheurs que chez les enseignants. Elles deviennent particulièrement utiles et stimulantes dans les études de cas sur les entrepreneurs. Elles permettent d'aller plus loin dans l'analyse des éléments de cohérence de chaque cas et se révèlent un outil précieux dans un domaine où il existe une telle diversité de cas. Le nombre de typologies développées est considérable; nous en avons retenu quelques-unes et la nôtre est présentée en annexe (Filion, 1988).

Cole (1959) a établi trois types d'opérations d'affaires : innovation, imitation et répétition. Collins, Moore et Unwalla (1964) et Collins et Moore (1970) ont établi une distinction entre l'« entrepreneur administratif » et l' « entrepreneur indépendant ». Smith (1967) a d'abord observé deux types d'entrepreneurs : l'entrepreneur artisan et l'entrepreneur opportuniste ou l'entrepreneur d'affaires. Il a considéré l'entrepreneur technologique comme faisant partie d'une catégorie à part. Smith et Miner (1983) ont ensuite regardé les implications de chaque type pour mieux comprendre le type d'entreprise associé à chacun. Lorrain et Dussault (1988b) ont analysé le comportement de gestion de chacun et trouvé que la gestion de l'entrepreneur opportuniste apparaît plus équilibrée. À partir de la recherche de Miner, Smith et Bracker (1989), Miner (1990) a montré des différences entre trois types: l'entrepreneur, l'entrepreneur axé sur une forte croissance et le manager. Laufer (1974) a relevé quatre types d'entrepreneur, soit : le manager ou l'innovateur, l'entrepreneur-propriétaire orienté vers la croissance, l'entrepreneur refusant la croissance mais recherchant l'efficacité, l'entrepreneur artisan. Glueck (1977) a établi une distinction entre trois types de travailleurs autonomes: l'entrepreneur, le propriétaire-dirigeant de PME, le dirigeant d'entreprise familiale. Gasse (1978), dans sa grille pour évaluer l'idéologie d'affaires et du management, a élaboré deux types d'idéologie entrepreneuriale : l'idéologie de l'entrepreneur artisan et celle de l'entrepreneur d'affaires. Chicha et Julien (1979) ont catégorisé les PME en trois types : traditionnelles, entrepreneuriales (orientées vers la promotion) et administratives (ou professionnelles). 
Scase et Goffee (1980) ont aussi établi des catégories d'entreprises. Schollhammer (1980) a établi cinq types d'entrepreneuriat corporatif : administratif, opportuniste, acquisitif, incubatif et imitatif. Pour Vesper (1980), il existe au moins 11 types d'entrepreneurs : 1) les travailleurs autonomes travaillant seuls, 2) les bâtisseurs d'équipes, 3) les innovateurs indépendants, 4) les multiplicateurs de modèles existants, 5) les exploitants d'économies d'échelle, 6) les rassembleurs de capitaux, 7) les acquéreurs, 8) les artistes qui achètent et vendent, 9) les constructeurs de conglomérats, 10) les spéculateurs, 11) les manipulateurs de valeurs apparentes. Filion (1988) a suggéré deux catégories de PME : la classique et la comète. Il a aussi proposé six types de propriétairesdirigeants de PME : le bûcheron, le séducteur, le sportif, le vacancier, le converti, le missionnaire (1988) [voir annexe 1] et deux types d'entrepreneurs : l'opérateur et le visionnaire (1996a). Ibrahim (1994) a établi une relation entre les types de stratégies et la performance des PME. S'appuyant sur Miles et Snow (1978), Julien et Marchesnay (1996), en tenant compte du critère de l'innovation, ont trouvé quatre types d'entrepreneurs : le prospecteur, l'innovateur, le suiveur et le réacteur. À partir de la logique d'action, ils ont défini deux types de propriétaires-dirigeants : le PIC (pérennité, indépendance, croissance) et le CAP (croissance forte, autonomie, peu de pérennité) [Julien et Marchesnay, 1987].

Julien (1990) a aussi proposé une typologie multicritère des PME. Après avoir présenté les critères sous-jacents aux principales typologies des PME décrites dans la littérature, il retient un ensemble de critères qualitatifs et quantitatifs placés sur un continuum en vue de définir une PME. Ces principaux critères sont: la taille, le secteur, le marché, le niveau d'indépendance et de centralisation, le type de stratégie et de technologie. Lafuente et Salas (1989) ont établi une typologie des nouveaux entrepreneurs ayant créé une entreprise en Espagne. Elle est basée sur leurs aspirations au travail et comprend quatre types: l'artisan, l'orienté vers le risque, l'orienté vers la famille et le managérial. Woo, Cooper et Dunkelberg (1991) ont évalué et discuté l'impact des critères utilisés sur la formulation des typologies d'entrepreneur.

Évidemment, il n'existe pas de typologie assez complète pour pouvoir classer tous les entrepreneurs et tous les propriétaires-dirigeants. À la limite, chaque cas est unique. Toutefois, une typologie permet de mieux comprendre les points d'ancrage, l'ensemble du système de valeurs et de pensée, et de créer des repères pour comprendre la cohérence d'ensemble du comportement d'un acteur.

\section{Tendances à théoriser}

On peut observer dans toute discipline un désir de comprendre des ensembles et de se donner des lois universelles autour desquelles on peut mieux organiser les connaissances. Les domaines de l'entrepreneuriat et de la PME n'y font pas 
exception. Nous avons jugé utile d'établir ici une distinction entre les deux domaines et d'aborder, dans un premier temps, sommairement, celui de la PME pour discuter par la suite de l'entrepreneuriat.

La Grande-Bretagne fut l'un des premiers pays où l'on réalisa l'importance de la PME pour la croissance économique. Après la Première Guerre mondiale, en 1919, on créa de multiples groupes de recherche pour mieux comprendre ce phénomène. Déjà, à cette époque, on avait décelé la tendance aujourd'hui bien connue que ce sont les PME qui créent l'essentiel de l'emploi (Birch, 1983 ; Peterson, 1977). On créa d'autres commissions d'études au cours de la crise économique des années 1930, puis en 1969, la commission Bolton, laquelle soumit son rapport en 1971. Le rapport révèle, entre autres, que des PME existent lorsque les économies d'échelle ne sont pas toujours possibles pour les grandes entreprises. Les PME voient aussi le jour parce qu'il existe des entrepreneurs et qu'un milieu favorable leur permet de créer des entreprises. Bolton (1971) et ses successeurs (Stanworth et al., 1982 ; Stanworth et Gray, 1991) offrent d'excellentes synthèses sur l'état des connaissances en PME qu'il serait trop long de résumer ici (voir Filion, 1992). Mentionnons, cependant, que le chapitre 7 de ce dernier livre présente une discussion intéressante sur l'état des connaissances sur le propriétaire-dirigeant. On y remarque qu'il n'existe pas de consensus quant aux caractéristiques comportementales du propriétaire-dirigeant de PME. Toutefois, les auteurs résument en disant qu'on a plus de chances de se lancer en affaires au Royaume-Uni si l'on est un homme, d'un âge moyen ou inférieur à l'âge de la retraite, si l'on vient d'un milieu familial où l'un des parents ou mieux les deux étaient engagés dans quelque activité d'affaires, si l'on est marié, si l'on a étudié une forme quelconque d'apprentissage et, finalement, si l'on appartient à un groupe ethnique provenant du sous-continent indien ou de la Méditerranée.

David J. Storey (1982) a beaucoup étudié les conditions qui président à la création et au développement des entreprises. Il propose l'équation suivante :

$\mathrm{E}=f(\pi, \mathrm{BE}, \mathrm{GR}, \mathrm{C})$

soit, $\mathrm{E}=$ entrée, $\pi=$ profits $(+), \mathrm{BE}=$ barrières à l'entrée $(-), \mathrm{GR}=$ croissance (growth) $(+)$,

$$
\text { C = concentration (-). (Storey, 1994, p. 61) }
$$

Là où existe une PME se trouve un entrepreneur qui l'a créée. En ce sens, le domaine de la PME, tout comme celui du travail autonome, constitue l'un des paramètres du champ plus vaste de l'entrepreneuriat. En entrepreneuriat, nous arrivons à un point où bien des gens voudraient voir apparaître une théorie robuste et solide basée sur quelques axiomes universels, comme c'est le cas en physique. Cette théorie serait basée sur des modèles quantitatifs rigoureux et découlerait de vastes recherches quantitatives démontrant de façon irréfutable 
la nature de l'entrepreneur et de ses activités, ainsi que leurs conséquences sur le développement économique. Par ailleurs, des milliers de professeurs sont aux prises chaque jour avec des besoins de matériel pour former des entrepreneurs en vue de la pratique. Pour ce faire, ils ont recours à des méthodes qualitatives en vue de développer des modèles et des outils qui permettent aux entrepreneurs actuels et potentiels d'exercer leur métier avec compétence. Cette tension entre universitaires qui se parlent et universitaires qui parlent à des praticiens de même qu'entre les deux groupes demeure suffisamment présente dans le champ pour qu'on la mentionne.

Les essais de théorisation sont nombreux. Retenons-en quelques-uns parmi les plus fréquemment cités : Amit, Glosten et al., 1993 ; Baumol, 1993 ; Bull et Willard, 1993 ; Bull, Thomas et Willard, 1995 ; Bygrave, 1989a et b, 1993 ; Casson, 1982 ; Collins, Moore et Unwalla, 1964 ; Collins et Moore, 1970 ; Covin et Slevin, 1991 ; Gartner, 1985, 1990 ; Gartner, Carland et al., 1988 ; Hébert et Link, 1982 ; Hofer et Bygrave, 1992 ; Leibenstein, 1968 ; Low et MacMillan, 1988 ; Peterson et Ainslie, 1988 ; Reynold, 1991 ; Sombart, 1928 ; Stevenson et Jarillo, 1990. Wortman et Birkenholz (1991) ont présenté une synthèse et un essai de classification d'un grand nombre de ces recherches. L'analyse des essais théoriques dans le champ de l'entrepreneuriat nous oblige à admettre avec Mulholland (1994) que l'association que Schumpeter (1928, 1934) a établie entre l'entrepreneur et l'innovation demeure dominante dans la discipline, en particulier chez les économistes. Nous avons mentionné précédemment dans ce texte l'invasion du champ de l'entrepreneuriat et l'appropriation par les diverses disciplines des sciences humaines et leur appropriation des éléments considérés pertinents par chacun en ce qui concerne l'entrepreneur. On peut d'ailleurs y voir la cause de l'apparition d'un nombre aussi varié de définitions et de bases aussi disparates quant à la façon d'aborder le sujet. Pour les économistes, la définition ainsi que l'approche de Schumpeter pour expliquer l'entrepreneur en relation avec l'innovation demeurent suffisantes pour élaborer une théorie de l'entrepreneuriat (Kirchhoff, 1992, 1994). Julien (1989) a d'ailleurs déjà souligné les difficultés d'arrimage de l'économie et des autres sciences humaines. En réalité, lorsqu'on compare les points de vue de Baumol (1990, 1993) et de Casson (1982), il est possible d'observer des différences fondamentales entre les économistes eux-mêmes.

Nous présentons dans les lignes qui suivent quelques auteurs qui ont réfléchi à la structuration ainsi qu'à la théorisation dans le champ de l'entrepreneuriat. Béchard (1996) suggère trois axes pour comprendre le champ de l'entrepreneuriat: praxéologique, disciplinaire et épistémologique. Selon Cunningham et Lischeron (1991), le champ de l'entrepreneuriat est en train de se structurer autour de six axes : école du grand homme, des caractéristiques psychologiques, classique (soit celle de l'innovation), du management, du 
leadership, de l'intrapreneurship. Reprenant ces caractéristiques avec d'autres, Blawatt (1995) a soutenu qu'un modèle conceptuel de l'entrepreneuriat doit inclure le critère de la performance. Il souligne que la majorité des modèles proposés à partir de l'école des traits, caractéristiques et autres théories sont la plupart du temps statiques. Il se situe dans la ligne de pensée d'auteurs qui ont étudié bon nombre d'entrepreneurs sur le terrain et qui ont observé qu'ils exploitent leur entreprise dans un contexte évolutif où activités et rôles sont appelés à se modifier graduellement. Ils apprennent de ce qu'ils font (Collins et Moore, 1970; Filion, 1988, 1989a et b, 1990a et b, 1991a et b), et comme la nature de ce qu'ils font évolue, eux aussi doivent évoluer. Ils sont ainsi appelés à apprendre à jouer des rôles différents au fur et à mesure de l'évolution de leur entreprise.

Deux des articles les plus stimulants à retenir pour théoriser en entrepreneuriat ont été écrits par un spécialiste du domaine qui possède la double formation doctorale en physique et en entrepreneuriat, en plus d'avoir œuvré comme scientifique et comme entrepreneur. Il s'agit de Bygrave qui a d'abord montré que l'entrepreneuriat devait se libérer du paradigme de la physique et des approches quantitatives pour trouver sa propre logique. Il est d'avis que ce dont on a le plus besoin, c'est de faire des recherches qualitatives sur le terrain pour mieux comprendre ce que font les entrepreneurs (1989a). Il a ensuite avancé (1989b) que la théorie du chaos en physique pourrait offrir une nouvelle façon d'aborder l'entrepreneuriat. Cependant, il met le chercheur en garde en soulignant que la théorie du chaos n'est «pas plus qu'une métaphore mathématique où l'exactitude des mesures nécessaires est inatteignable pour expliquer le processus» (1993).

Après avoir analysé les thèmes traités et les références données dans une des revues fréquemment citées du champ, le Journal of Business Venturing, Déry et Toulouse (1994) ont observé que plus de $50 \%$ des références proposées provenaient de livres. Une recherche semblable, réalisée en stratégie par l'analyse des citations dans le Strategic Management Journal, a montré que plus de $50 \%$ des références provenaient d'articles universitaires. Cela serait une indication que la discipline de la stratégie arrive à un point suffisant de maturité où il existe des consensus entre chercheurs. En entrepreneuriat, nous serions encore, selon eux, dans un paradigme en construction où il n'existe pas de consensus quant à la construction théorique du domaine. On peut aussi émettre l'hypothèse que la construction théorique du champ de l'entrepreneuriat est en train de se réaliser de façon différente de ce que nous avons observé jusqu'ici pour les autres disciplines des sciences humaines, y compris la stratégie. Alors que la psychologie est issue de la philosophie (Miller, 1962), la psychanalyse de la médecine et de la psychologie, les racines du champ de l'entrepreneuriat sont multiples et proviennent de presque toutes les disciplines des sciences humaines 
et administratives. Les recherches touchent tant des éléments de théorie que de pratique. Il ne serait pas surprenant de voir apparaître des théories qui découlent d'ensembles de recherches appliquées. En effet, des rencontres avec de nombreux chercheurs du domaine nous font réaliser que ceux qui y œuvrent y sont sur-sollicités pour développer cours, programmes et recherches appliquées de telle sorte que, même s'il existe un intérêt pour la théorie, peu de gens ont en réalité du temps à consacrer à son élaboration. Celles et ceux qui comptent le faire devront être imaginatifs et ne pas penser à une approche unidimensionnelle comme c'est le cas dans de nombreuses sciences. Faut-il rappeler que les sciences de l'homme sont essentiellement composées de modèles interprétatifs souples et qu'une théorie de l'entrepreneuriat se devra d'être souple et multidimensionnelle afin de bien refléter sa multidisciplinarité.

\section{Définition}

On ne saurait présenter un texte synthèse sur l'entrepreneuriat sans y définir le terme. Il est intéressant d'observer que certains des mots clés utilisés dans le langage moderne des sciences administratives viennent du français. Par exemple, le terme «manager » vient du vieux français «ménager», qui voulait dire tenir maison avec parcimonie, s'organiser. Définir l'entrepreneur constitue toujours un défi, compte tenu de la très grande variété de perspectives à partir desquelles les spécialistes qui ont étudié l'entrepreneur l'ont fait. Cochran (1968) a noté que, pour les économistes en particulier, l'entrepreneur représente une « incongruité d'un élément humain non mesurable dans une structure théorique ». En fait, quelle que soit la définition que l'on donnera, il risque de se trouver quelqu'un qui ne s'y retrouvera pas. La définition que nous allons proposer se veut un dénominateur commun assez large pour que le plus grand nombre puisse s'y retrouver. Cette définition tient compte des grands courants de la littérature en entrepreneuriat. Rappelons qu'Hélène Vérin a étudié l'évolution du terme « entrepreneur » à travers l'histoire. Il est intéressant d'observer qu'au XII ${ }^{\mathrm{e}}$ siècle, le terme référait à un souteneur «de mauvaise querelle» (Vérin, 1982, p. 31). $\mathrm{Au} \mathrm{XVII}{ }^{\mathrm{e}}$ siècle, le terme référait à quelqu'un qui engage et dirige une action militaire. Ce n'est que depuis la fin du XVII ${ }^{\mathrm{e}}$ siècle que le terme signifie une personne qui «forme et réalise un projet» $(1982$, p. 33) ou qui «créé et dirige une entreprise » (1982, p. 32). À l'époque de Cantillon, là où le terme va prendre vraiment la signification qu'on lui donne aujourd'hui, on utilisera ce terme pour désigner quelqu'un qui achète des matières premières, les transforme et les revend à quelqu' un d'autre. Il s'agit donc d'un intermédiaire qui a identifié une occasion d'affaires, qui a pris un risque en décidant de transformer une matière première acquise et de la revendre. Donc, la dimension de risque apparaît dans les observations qu'on aura sur ce que fait l'entrepreneur dès le début du XVIII ${ }^{\mathrm{e}}$ siècle. La définition que nous proposons représente autant une description qu'une 
interprétation de ce qu'est et de ce que fait l'entrepreneur. Elle tient compte de l'étude d'une soixantaine de définitions parmi les plus couramment utilisées dans la littérature (Filion, 1987 et 1988). Elle se place dans l'optique de Pinchot (1986) lorsqu'il écrit que les intrapreneurs sont des «rêveurs qui passent à l'action ». Comme plusieurs autres, Lynn (1969) a montré le parallèle qui existe entre les entrepreneurs et les créateurs. Nous tenons aussi compte de cette dimension que nous considérons fondamentale pour comprendre le comportement de l'entrepreneur, c'est-à-dire une personne créative et dont l'imagination demeure très active. Nous avons observé que cette imagination fonctionne à au moins deux niveaux : l'entrepreneur peut imaginer la situation, le scénario dans lequel il va s'engager et à partir duquel il va créer son entreprise, mais par la suite il imagine aussi un nombre considérable d'autres manières de s'organiser et d'agir pour arriver à réaliser son projet, sa vision. La première partie de notre définition se lira donc de la façon suivante :

«Un entrepreneur est une personne imaginative... »

Cette personne imaginative aime se fixer des buts qu'elle sait pouvoir atteindre. Ils ne sont pas toujours écrits, mais ils sont là, et ils constituent un fil conducteur, une vision autour de laquelle l'entrepreneur organise ses activités. C'est pour réaliser son but qu'il entre en action et qu'il développe les caractéristiques de ténacité, d'internalité, de créativité, qu'on lui a souvent attribuées dans la recherche.

Le second élément de notre définition exprime donc cette dimension:

«... caractérisée par une capacité à se fixer et à atteindre des buts. »

Une autre dimension très présente dans la littérature consiste à associer l'entrepreneur avec l'identification d'occasions d'affaires, avec la capacité de détecter des occasions d'affaires. Cela implique que l'entrepreneur développe une très grande sensibilité par rapport à son environnement. Nous exprimons cette dimension de la façon suivante:

"Cette personne maintient un degré élevé de sensibilité en relation avec son environnement en vue d'y déceler des occasions d'affaires.»

En fait, tant qu'elle continuera à déceler des occasions d'affaires et à passer à l'action pour les exploiter, cette personne continuera à jouer un rôle entrepreneurial. En réalité, l'entrepreneur est une personne qui doit continuer à apprendre. Non seulement continuer à apprendre par rapport à ce qui se passe dans son environnement afin d'y déceler des occasions d'affaires, mais continuer à apprendre de ce qu'il fait et à s'ajuster en conséquence dans son entreprise. En fait, tant et aussi longtemps qu'il continue à apprendre, l'entrepreneur continue à jouer son rôle; il continue à passer à l'action. Il est dans un processus en continuelle évolution. Mais l'objet premier de cet apprentissage, pour que 
le rôle entrepreneurial se perpétue, demeure toujours le repérage d'occasions d'affaires. Nous l'intégrons dans notre définition de la façon suivante:

"Aussi longtemps qu'il ou elle continue d'apprendre au sujet d'occasions d'affaires possibles...»

Un entrepreneur n'a pas le comportement de quelqu'un qui joue à la roulette. C'est quelqu'un qui met en place des projets, le plus souvent des projets d'affaires. Pour réussir, il devra minimiser le plus possible le niveau de risque qui entoure ses décisions. La recherche montre que l'entrepreneur est quelqu'un qui tend à prendre des risques modérés, à minimiser l'incertitude dans son processus de prise de décisions. Nous exprimons cette dimension de la façon suivante :

«... et qu'il ou elle continue à prendre des décisions modérément risquées... »

De plus, depuis Jean-Baptiste Say et Joseph Alois Schumpeter, on a beaucoup associé l'entrepreneur à l'innovation. L'entrepreneur est quelqu'un qui est un agent de changement, qui fait des choses nouvelles et différentes. On ne saurait concevoir un entrepreneur qui n'apporterait pas quelque chose de nouveau. Nous avons intégré cette dimension de la façon suivante :

«... qui visent à innover, il ou elle continue de jouer un rôle entrepreneurial.»

Cette définition se lit comme suit :

«Un entrepreneur est une personne imaginative, caractérisée par une capacité à se fixer et à atteindre des buts. Cette personne maintient un niveau élevé de sensibilité en vue de déceler des occasions d'affaires. Aussi longtemps qu'il ou elle continue d'apprendre au sujet d'occasions d'affaires possibles et qu'il ou elle continue à prendre des décisions modérément risquées qui visent à innover, il ou elle continue de jouer un rôle entrepreneurial. » (Filion, 1988)

Nous pourrions présenter une définition beaucoup plus succincte qui la résume et en exprime l'essentiel. Elle se lirait de la façon suivante :

«Un entrepreneur est une personne qui imagine, développe et réalise ses visions. » (Filion, 1991b)

Cette définition inclut les éléments qui sont présentés dans la définition précédente, en ce sens que pour imaginer une vision, cela implique que l'entrepreneur soit une personne imaginative. Le terme « vision » implique qu'on soit capable de se fixer des buts et de les atteindre. La différence entre le rêve et la vision réside dans le fait que la vision constitue une forme de rêve, mais réaliste et réalisable. C'est une image désirée d'un état futur. Cela implique aussi qu'on maintienne un niveau élevé de sensibilité en relation avec l'environnement en vue d'y déceler des occasions d'affaires. Pour que la vision puisse se développer, il faut que l'entrepreneur continue à apprendre de son 
environnement. Il doit aussi, pour la réaliser, prendre des décisions modérément risquées s'il veut rester en affaires. Ces décisions doivent inclure des dimensions nouvelles dans ce qui est fait. Une vision implique qu'on présente quelque chose de nouveau, qui soit motivant pour les gens de l'organisation et attrayant pour le marché. Tant que l'entrepreneur continue à imaginer, à développer et à réaliser des visions qui constituent le fil conducteur autour duquel il organise ses activités, il continue à jouer un rôle entrepreneurial. Quelqu'un qui a fait une invention sera considéré comme un inventeur toute sa vie. Quelqu'un sera généralement considéré comme entrepreneur tant et aussi longtemps qu'il continue à jouer un rôle entrepreneurial. On dira de quelqu'un qui a vendu son entreprise qu'il était entrepreneur.

Il faut cependant établir une distinction entre l'entrepreneur et le propriétaire-dirigeant de PME. En effet, plusieurs personnes exerceront des rôles entrepreneuriaux sans devenir pour autant des propriétaires-dirigeants de PME ; soit qu'ils exercent leurs activités entrepreneuriales à l'intérieur de grandes entreprises et qu'ils jouent un rôle d'intrapreneur, soit qu'ils lancent une entreprise ou qu'ils opèrent comme travailleur autonome sans pour autant développer une petite entreprise. À l'opposé, nous avons plusieurs propriétairesdirigeants qui ont acquis une entreprise sans l'avoir créée et qui n'ont pas apporté de changements significatifs et qui n'ont pas une vision de ce qu'ils veulent faire, qui n'ont pas développé ni de nouveaux produits ni de nouveaux marchés, et qui gèrent au jour le jour, prenant des décisions au sujet d'activités de gestion courantes, mais sans avoir ni de vision, ni de plan d'ensemble, ni de but précis bien articulé. On ne peut qualifier ces propriétaires-dirigeants d'entrepreneurs : ce sont des propriétaires-dirigeants de PME qui ne jouent pas un rôle particulièrement entrepreneurial.

Toute définition présente aussi des limites reliées à notre perception et à notre compréhension du sujet ; mais il était, à notre avis, indispensable, dans un texte comme celui-ci, de définir au moins les termes clés.

\section{Réflexions et prospectives}

Les années 1980 voient l'éclosion du domaine et son intégration dans de nombreuses disciplines. L'accélération de la vitesse du changement technologique pousse les organisations et les sociétés à rechercher de nouvelles approches pour mieux intégrer ces changements dans leur dynamique. On a compris, avec le plafonnement du développement dans les pays communistes, qu'une société peut difficilement se développer sans entrepreneurs. La principale ressource d'une société est la ressource humaine, mais elle doit être mise en mouvement par et autour de projets entrepreneuriaux. Après la chute de l'URSS, ce mouvement vers la performance - suivre ou devancer l'autre - semble s'accentuer (Fukuyama, 
1992). Selon cet auteur, la prospérité et ce qui la sous-tend, l'entrepreneuriat, serait la résultante d'états de confiance qui existent entre personnes dans une société (1994).

Non seulement la recherche en entrepreneuriat s'étend-elle à la plupart des disciplines, mais le nombre d'institutions qui offrent des cours dans le domaine et le nombre de cours offerts ont plus que doublé en une décennie (Vesper, 1985, 1993). Comme dans plusieurs pays, on voit apparaître au Québec, au début des années 1980, des galas régionaux annuels d'entreprises lors desquels des dizaines de prix sont remis aux entreprises les plus performantes : meilleur entrepreneur de l'année, meilleur marketing, meilleure exportation, meilleure protection de l'environnement, meilleure gestion des ressources humaines, etc. Les gagnants régionaux seront présentés au concours provincial, national, puis international. C'est la glorification de l'entrepreneur et la reconnaissance de modèles à proposer à la société pour prospérer.

Au-delà de cela, il faut voir dans l'entrepreneuriat une nouvelle étape vers l'acquisition de la liberté par un plus grand nombre. Il se peut que la société entrepreneuriale actuelle soit en mutation profonde et que l'entrepreneuriat soit amené à s'exprimer dans des formes organisationnelles de plus en plus réduites. Ainsi, on serait passé de l'expression de l'entrepreneuriat dans les grandes organisations au début du siècle avec un continuum en diminution graduelle amorcé vers les années 1970 . On a vu l'entrepreneuriat prendre forme dans les PME depuis les années 1920 pour privilégier cette forme à compter des années 1970. Depuis la décennie 1990, on a observé une proportion de plus en plus grande de l'entrepreneuriat se manifester par le travail autonome.

On voit apparaître dans les diverses formes d'entrepreneuriat des volontaires et des involontaires (Filion, 1996). En fait, l'apparition des involontaires est un phénomène de la décennie de fin de siècle. Ce sont les diplômés ainsi que les mis à pied à la suite de fermetures et de restructurations d'entreprises qui n'ont pas réussi à se trouver d'emploi et qui doivent se lancer en affaires pour s'en créer un. Leur formation ainsi que leur préparation nécessitent des approches fort différentes de celles que nous avons utilisées jusqu'ici pour les volontaires. Ils tendent à privilégier le travail autonome comme forme entrepreneuriale, mais ce ne sont pas des entrepreneurs au sens où on l'entend généralement. Ils créent une activité d'affaires, mais ne sont pas pour autant attirés vers l'innovation. Alors que les mots clés qui définissent l'entrepreneur incluent la nouveauté et la croissance, les mots clés qui définissent le travailleur autonome sont plutôt l'écologie personnelle et l'équilibre de vie.

Un autre phénomène qu'il est possible d'observer et qui suscite l'apparition de formes entrepreneuriales de plus petite dimension réside dans le changement qu'est en train de subir la notion de succès (Filion, 1996). On observe, en effet 
que la notion de succès tend à prendre en considération, chez les jeunes entrepreneurs plus instruits, davantage des critères intrinsèques reliés à la réalisation de soi que des critères extrinsèques reliés aux performances de l'entreprise et au niveau de statut atteint. Cet élément explique aussi la tendance vers la formation de plus petites unités.

En réalité, la vitesse du changement s'accélère et rend la gestion des grandes organisations de plus en plus difficile. Il faut que l'apprentissage (A) et sa mise en pratique $(\mathrm{P})$ de façon créative $(\mathrm{Cr})$ soient plus grands que la vitesse du changement $(\mathrm{C})$ pour qu'une personne, une organisation, une société s'adapte et suive le rythme d'évolution de son environnement. En réalité, cette formule pourrait exprimer les éléments les plus fondamentaux de l'entrepreneuriat :

$$
\text { [( } \mathrm{A}+\mathrm{Cr}) \mathrm{P}>\mathrm{C}]
$$

L'expression et la réussite de la pratique entrepreneuriale sont fonction de plusieurs éléments dont deux ont été très peu abordés en entrepreneuriat: la gestion de l'espace et du temps. L'entrepreneur doit apprendre à gérer des espaces internes et externes à son entreprise. Il doit apprendre à identifier, puis à définir et à délimiter un espace à occuper dans le marché. Comme la plupart des domaines d'activités d'affaires sont cycliques, la définition de l'espace et du temps deviendront aussi des éléments clés pour expliquer la réussite, par exemple, dans la gestion des stocks et des liquidités. Il doit ensuite définir un espace organisationnel physique ainsi que des espaces psychologiques individuels aux collaborateurs, la variation de cet espace dépendant de la perception des capacités et des compétences qu'entretient l'entrepreneur à l'égard de chacun. D'ailleurs, sa façon de gérer les espaces aura d'énormes conséquences sur le temps. Plus il aura visé juste dans l'attribution des espaces individuels de même que dans le choix des espaces à occuper dans le marché, plus il aura de temps à consacrer à la détermination d'autres espaces.

Tout en gérant des espaces, l'entrepreneur doit gérer le temps. Le temps qu'un espace demeure disponible dans le marché devient de plus en plus court. De même, la vitesse accélérée du changement rendra les ajustements que chacun doit apporter à sa façon de faire les choses d'autant plus faciles qu'on aura laissé à chacun un espace individuel plus large. Plus les gens auront intériorisé la culture et les règles de l'organisation, plus on leur fera confiance, plus on leur accordera d'espace. On peut assumer que plus ils ont d'espace, plus ils sont en mesure d'opérer rapidement, car le nombre de personnes à qui ils doivent avoir recours pour faire ce qu'ils font sera d'autant plus limité.

En fait, on est arrivé à un point où la vitesse même du changement technologique est reliée à la capacité des individus et des organisations à gérer de manière entrepreneuriale, c'est-à-dire avec créativité et rapidité. Il est peu 
probable qu'on revienne à une époque où la majorité des gens d'une entreprise effectueraient pendant des années les mêmes tâches répétitives. En réalité, il est peu probable qu'on voie se maintenir pendant des années dans une situation de leadership des entreprises qui emploieront plus de 5000 personnes, réparties dans des unités de plus de 200 personnes. Cela se comprend à partir du corollaire que plus l'organisation est grosse, plus elle met du temps à apprendre et à changer, et qu'au-delà d'un certain nombre de personnes, le temps requis pour le changement interne est plus grand que la vélocité du changement externe.

En ce sens, l'ère qui s'annonce en est une où l'entrepreneuriat sera florissant. Mais plus la vitesse du changement technologique continuera d'augmenter, plus l'entrepreneuriat aura tendance à s'exprimer sous de plus petites formes organisationnelles. C'est là la résultante de l'application de la formule présentée quelques paragraphes plus haut ; la relation est inversement proportionnelle. Les entreprises qui réussiront et croîtront auront énormément recours à la sous-traitance et à diverses formes de franchisage. L'entrepreneuriat se développera suivant toutes sortes de formes nouvelles comme, par exemple, par le travail autonome tant dans des réseaux pairs que complémentaires (Filion, 1996b). La marche des sociétés vers sa prise en charge par chacun, qu'elle soit volontaire ou involontaire, marque une nouvelle étape vers l'acquisition d'une plus grande liberté, par un plus grand nombre. C'est là que la recherche en entrepreneuriat prend tout son sens, car elle offre des outils à un nombre de plus en plus grand de personnes pour leur permettre d'agir suivant leurs propres termes, d'être eux-mêmes dans l'action et de partager cette expérience avec d'autres.

\section{Conclusion}

Nous avons vu que l'entrepreneuriat a d'abord été identifié par les économistes comme un phénomène utile pour mieux comprendre le développement. Les behavioristes ont, eux aussi, tenté de mieux connaître l'entrepreneur. Mais le champ subit un éclatement en ce sens qu'il est en train d'être intégré dans presque toutes les disciplines des sciences humaines.

Nous observons avec Mulholland (1994), Rosa et Bowes (1990) que le champ de l'entrepreneuriat demeure dominé par les positivistes-fonctionnalistes et qu'il existe un grand besoin d'ouvrir encore des perspectives nouvelles pour mieux comprendre l'ensemble de ce qu'est et de ce que fait l'entrepreneur. Aussi, nous nous devons de mentionner les travaux remarquables d'un chercheur qui a ouvert une piste de recherche originale, par la cartographie cognitive, pour mieux comprendre la logique stratégique de l'entrepreneur: Pierre Cossette (1994a et b). Il pourrait aussi être intéressant de pousser davantage les recherches du côté de la vision entrepreneuriale (Filion, 1991a et b), ainsi 
que sur le concept et l'espace de soi perçus, développés et acquis par l'entrepreneur (Filion, 1993, 1994).

Compte tenu de ce qui a été écrit dans ce cahier, nous pouvons définir l'entrepreneuriat comme étant le champ qui étudie la pratique des entrepreneurs : leurs activités, leurs caractéristiques, les effets économiques et sociaux de leur comportement ainsi que les modes de soutien qui leur sont apportés pour faciliter l'expression d'activités entrepreneuriales. Tout domaine d'études ne peut négliger la théorie. Pour vraiment théoriser sur l'entrepreneur, il faudra probablement parvenir à dissocier les recherches appliquées des recherches théoriques et établir une nouvelle science : «l'entreprenologie». Celle-ci pourrait intégrer dans un corpus théorique les convergences issues des études théoriques menées par les «entreprenologues» sur les entrepreneurs dans l'ensemble des disciplines. L'entrepreneuriat continuerait de rassembler l'ensemble des recherches appliquées qui intéressent les entrepreneurs potentiels et actuels. Il nous faudra peut-être attendre encore quelques milliers de publications et quelques décennies pour en arriver là.

\section{ANNEXE I}

\section{Six types de propriétaire-dirigeant de PME}

Nous retrouvons six types de proprio-dirigeant de PME:

- le bûcheron (lumberjack);

- le séducteur (seducer)

- le sportif (player);

- le vacancier (hobbyist);

- le converti (convert);

- le missionnaire (missionnary).

\section{Le bûcheron}

Il n'aime pas la foule. Lorsqu'il parle avec quelqu'un, il a l'impression de perdre son temps. Il est ambitieux et possède de bonnes capacités de travail. Il aime faire des choses. En fait, lorsqu'il travaillait en grande entreprise ou pour les autres, il accomplissait le double de la quantité du travail de n'importe qui d'autre. C'est là qu'il a acquis la conviction que pour recevoir son véritable dû, il se devait d'aller travailler pour lui-même. Il aime aiguiser sa scie et couper du bois. Il le fait bien, même mieux que beaucoup d'autres. Il le fait de l'aube au crépuscule. 
Lorsque les gens qui achètent ses produits en veulent plus - en particulier à cause de leur qualité - il engagera du personnel et leur fera couper du bois. Il sera la plupart du temps insatisfait tant de la qualité que de la quantité de travail accompli par les gens qu'il aura engagé. Il préfère ceux qui travaillent dur et bien comme lui. La culture organisationnelle est axée sur la production.

Il se peut qu'à un certain moment de sa carrière, il cesse de ne regarder que les arbres pour jeter un coup d'œil à la forêt. S'il atteint ce premier niveau de pensée stratégique, l'entreprise qu'il dirige pourra peut-être vraiment commencer à croître. On s'attaquera le plus souvent au marché des produits potentiels connexes, et c'est à partir de là qu'on développera.

Le bûcheron demeure le type le plus courant de proprio-dirigeant de PME. Certains bûcherons qui auront réussi deviendront éventuellement des missionnaires.

\section{Le séducteur}

Il aime s'impliquer totalement, mais cela ne dure pas longtemps. Il lance les entreprises et les vend. Il reprend des entreprises en difficultés et les revend. Il achète des entreprises, y coupe les coûts d'opération, y apporte quelques changements clés et les vend. Il aime que les choses se fassent rapidement. Il peut évaluer rapidement aussi bien les forces et les faiblesses que le marché potentiel d'une entreprise. C'est un type sociable qui compte beaucoup de gens parmi ses relations. Lorsqu'il regarde une entreprise en difficultés, il pense à une de ses relations qui pourrait acheter telle partie de la production, à une autre à qui l'on pourrait sous-traiter de la production, à une autre à qui l'on pourrait sous-traiter la production de telle composante, à une autre qui a mis sur pied une méthode pour réduire les coûts de distribution dans un des secteurs d'opération concerné, à une autre qui achèterait des quantités appréciables d'un des produits concernés s'il n'était que légèrement modifié, etc.

Mais les choses doivent continuer à bouger, autrement tout perd de l'intérêt et il commence à regarder ailleurs. Et vu que c'est un type qui rencontre beaucoup de gens, les occasions sont multiples. Tout ce qu'il regarde est vu à partir d'un point de vue bien précis : où et comment tirer un bénéfice au prix du moindre effort. Lorsqu'il songe à des entreprises, on peut détecter des signes de «piastres » dans ses yeux. Et derrière ses yeux, un petit ordinateur calcule continuellement ce que pourraient être les profits si on procédait à tel ou tel réajustement dans l'entreprise qu'on est en train de visiter.

Le séducteur ressemble à certains artistes en relation de leur art: son style, ses goûts, ses intérêts vont changer avec le temps. Il demeure cependant stimulé par ce qui est différent. Lorsqu'il vieillira, s'il décide de se brancher dans une entreprise, il risque fort de devenir un sportif. 


\section{Le sportif}

Il aime le loisir. Il regarde la pratique d'au moins un sport comme une activité vitale pour lui. Celui auquel il accorde le plus de temps variera au cours des années. Cela sera aussi lié à l'évolution de son système de relations.

Il voit son entreprise comme un support financier, comme un moyen qui lui permet de gagner les ressources suffisantes pour vaquer à ces autres activités qui l'intéressent vraiment. Souvent, il est dans un domaine cyclique, ce qui l'amènera à travailler fort pendant certaines périodes de l'année. Toutefois, il ne se sent pas impliqué émotivement dans ce qu'il fait, comme c'est le cas du converti, par exemple. Il tente de délimiter ce qu'il fait à ce qui rapporte, à ce qui est payant pour pouvoir profiter au maximum de ces autres activités qu'il aime vraiment mais qui l'obligent à travailler s'il veut être en mesure de continuer à les pratiquer. L'entreprise est vue comme un moyen, parfois même une contrainte nécessaire, qui lui permet de faire ce qu'il aime vraiment.

Souvent, il vient d'un milieu aisé où une bonne partie de sa jeunesse a été passée à pratiquer des sports ou à s'accorder du temps de loisir. Parfois, il en est à la troisième ou quatrième génération de propriété familiale de l'entreprise.

Un certain nombre de sportifs remplaceront graduellement la pratique de certains de leurs sports par des activités sociales et politiques.

\section{Le vacancier}

Il consacre toute son énergie et tous ses temps libres à son entreprise. Il a un autre emploi, officiel celui-là, mais il ne le conserve que par besoin de sécurité et parce qu'il apporte un support à son entreprise. Son entreprise, c'est le « hobby », le violon d'Ingres, le passe-temps par excellence. C'est là où il voit les possibilités de réaliser ses aspirations. C'est pourquoi il y investit toutes les ressources dont il dispose et qu'il peut trouver afin de la développer le plus possible.

Le vacancier demeure divisé entre des activités qui impliquent des logiques de fonctionnement fort différentes. Dans son «emploi officiel », il opère à un niveau relativement bas ou tout au plus intermédiaire. Il n'a pas à trouver des solutions ni à prendre des décisions qui sont d'un haut niveau de complexité.

À cause de cette conjoncture, son expérience ne lui apprend pas à établir des distinctions entre les différents niveaux de décisions, ce qui en fait un proprio-dirigeant qui aura longtemps des difficultés à bien articuler ses décisions stratégiques, car il est conditionné, par son activité principale de tous les jours, à prendre des décisions administratives.

En fait, il lui faudra beaucoup de temps avant d'arriver à prendre une des premières décisions stratégiques importantes: celle de quitter son «emploi 
officiel » afin de se consacrer entièrement à son entreprise. Et il continuera à évoluer pendant des années contre ces deux logiques de fonctionnement et de niveaux différents : celle de son «emploi officiel» et celle de la direction de sa petite entreprise.

Certains vacanciers deviendront des bûcherons, d'autres des convertis, mais tous finiront par montrer des tendances du sportif, car ils auront acquis un mode de fonctionnement double ou multiple, lequel implique une activité en vue d'en soutenir une autre, celle où l'on se réalise vraiment.

\section{Le converti}

Il l'a trouvée "l'affaire»! Par conséquent, tout dans sa vie découle de cette découverte fondamentale. La plupart du temps, cela représente le début d'une nouvelle carrière pour lui. Cela faisait des années qu'il recherchait quelque chose, ce quelque chose qui lui permettrait de vraiment se réaliser, d'utiliser son potentiel dans un sens vraiment positif. Il l'a finalement trouvé, c'est maintenant une obsession.

Attention aux commentaires que vous lui ferez sur cette nouvelle entreprise qu'il vient de lancer ou d'acquérir : il s'agit là de quelque chose de sacré, de quelque chose dans lequel il est déjà profondément impliqué de façon émotionnelle. Il s'est bâti une logique d'une cohérence rigoureuse qui explique tout ce qui s'y fait. Cette logique semble entourer «l'affaire » d'un halo protecteur. Infidèles, croyants en d'autres religions ou même, vous, observateurs curieux, demeurez circonspects dans vos remarques. Vous pourriez rapidement devenir l'objet de ses «foudres célestes ».

En fait, le converti en est rapidement venu à regarder le monde comme divisé en pour et en contre : d'une part, ceux qui aiment et qui approuvent ce qu'il fait et, d'autre part, tous les autres. Il tend à survaloriser ceux qui font et pensent comme lui et il demeure méfiant à l'égard des autres : ce ne sont peutêtre pas des gens totalement honnêtes... puisqu'ils ne pensent pas comme lui. Qui sait ce qu'on trouverait si on cherchait un peu dans leur vie... ce qu'on pourrait trouver à leur reprocher. En tous les cas, ils n'ont pas encore trouvé, eux. Ils ne savent pas ce qu'ils ratent.

Il ressent un véritable plaisir à faire des choses plus qu'à en observer les résultats, parce que dans tout ce qu'il fait, il est convaincu qu'il est en train d'accomplir une étape, si petite soit-elle, pour permettre à l'humanité de s' améliorer.

Il se voit comme quelqu'un doté de qualités particulières qu'il doit mettre au service de la société. Il aime être au contrôle. C'est ce qui l'amène à ne déléguer qu'avec difficulté, du moins tant que l'autre n'a pas vraiment fait ses preuves. 
Plusieurs créateurs et inventeurs tombent dans cette catégorie. En fait, les convertis qui viennent de la recherche et développement et du marketing / vente semblent ceux qui réussissent le mieux. Plusieurs finiront comme missionnaire.

\section{Le missionnaire}

La plupart du temps, il a lancé son entreprise lui-même, sinon il l'a transformé substantiellement. Il connaît très bien son produit ainsi que son marché. Il est dévoré par une véritable passion de ce qu'il fait. Il est convaincu que ce qu'il fait constitue un élément important pour la communauté dans laquelle il exerce son activité. D'une certaine façon, on peut dire qu'il est un converti qui a acquis un certain degré de maturité et qu'il est moins impliqué émotivement. Il a réussi à couper assez tôt le cordon ombilical qui le reliait à son entreprise et voit davantage les choses dans leur ensemble.

En fait, il a organisé l'entreprise assez rapidement, et le plus souvent lorsqu'elle était encore petite, de telle sorte qu'elle puisse fonctionner sans lui ; ou du moins sans qu'il y soit présent tous les jours.

Depuis que l'étape de survie a été dépassée, il ne se sent plus aussi menacé qu'il l'était et il est devenu beaucoup plus ouvert à toutes sortes de nouvelles idées. Il regarde l'entreprise comme un organisme vivant où l'apprentissage et l'évolution des membres présentent une dimension importante pour lui : il faut qu'ils y soient heureux. Son avenir est intimement lié à l'évolution de l'entreprise elle-même et, par conséquent, à sa capacité de demeurer compétitive et de se développer. Il reste aussi intimement lié à l'évolution harmonieuse de chacun de ses membres, car il a compris qu'une entreprise est un système social. Les résultats ne dépendent pas seulement des performances individuelles, mais de la capacité des gens à travailler ensemble.

Bien qu'il soit très orienté vers la tâche, il devient de plus en plus, au fil des ans, quelqu'un de préoccupé par les relations humaines. Presque tout son temps et ses énergies sont consacrés à l'entreprise. Conservateur dans ses valeurs, stable dans sa vie personnelle le plus souvent axée sur sa famille, très réaliste dans la pratique des affaires, ce qui l'intéresse, ce n'est pas surtout de développer une entreprise mais de bâtir une équipe - souvent à l'image de la famille - de construire un tissu social où l'on peut voir une culture organisationnelle ouverte où les gens se réalisent, progressent et où les échanges entre les gens font que l'organisation apprend.

Le missionnaire délègue tout ce qu'il peut. Il cherche à consacrer l'essentiel de son temps à prêcher, à communiquer, à participer à des groupes de travail où il peut écouter, discuter, échanger, enfin, demeurer un stimulateur qui apporte 
santé et bon sens à la vie de groupe. Il prêche par l'exemple. Il communique l'enthousiasme à ceux qui l'entourent. Ceux-ci le lui rendent bien, car ils demeurent hautement motivés, et font que l'entreprise remporte succès après succès. Elle deviendra bientôt une multinationale et «il faudra » aller porter la «bonne nouvelle» ailleurs. Avis aux membres : si vous ne voulez pas faire partie de cette religion ou si vous avez des réticences à vous y impliquer totalement, allez travailler ailleurs.

On trouvera ci-dessous le type de stratégie qui apparaît le plus logique pour chaque type de proprio-dirigeant proposé.

TABleau 1

Type de proprio-dirigeant de PME et stratégie

\begin{tabular}{lll}
\hline $\begin{array}{l}\text { Type } \\
\text { de proprio-dirigeant }\end{array}$ & $\begin{array}{l}\text { Raison d'être } \\
\text { de l'entreprise }\end{array}$ & $\begin{array}{l}\text { Type } \\
\text { de stratégie }\end{array}$ \\
\hline Bûcheron & survie-réussite & continue \\
Séducteur & plaisir & radicale \\
Sportif & loisirs & rationnelle \\
Vacancier & réalisation de soi & évolutive \\
Converti & sécurité & révolutionnaire \\
Missionnaire & conquête & progressive \\
\hline
\end{tabular}

Peu de types existent à l'état pur et comme pour toute typologie, les propriétaires-dirigeants rencontrés dans la vraie vie présentent souvent un profil mixte de deux ou trois types.

Nous avons élaboré cette typologie après avoir effectué une analyse empirique approfondie sur le terrain d'une centaine de cas de propriétairesdirigeants de PME (Filion, 1996b). Entre 1988 et 1996, cette typologie a été utilisée pour des études de cas en classe : environ 10 cas par an. Ainsi, plus de 80 propriétaires-dirigeants ont été classés à partir de cette typologie. En fait, elle permet d'établir le système de valeurs et d'intention de la personne concernée et de mieux comprendre ses modes de prises de décisions, ses orientations stratégiques ainsi que l'élaboration de son processus visionnaire. Il est intéressant d'observer que certains propriétaires-dirigeants évolueront d'un type à l'autre en cours de carrière. 


\section{Bibliographie}

Amit, R., L. Glosten et al. (1993), "Challenges to theory development in entrepreneurship research », Journal of Management Studies, vol. 30, $\mathrm{n}^{0} 5$, p. 815-834.

BANNOCK, G. (1981), The Economics of Small Firms: Return from the Wilderness, Oxford, Basil Blackwell.

BAUMOL, W.J. (1968), «Entrepreneurship in economic theory », The American Economic Review, $\mathrm{n}^{0}$ 58, p. 64-71.

BAUMOL, W.J. (1990), «Entrepreneurship : productive, unproductive, and destructive», Journal of Political Economy, vol. 98, n ${ }^{0}$ 5, p. 893-921.

BAUMOL, W.J. (1993), «Formal entrepreneurship theory in economics : existence and bounds », Journal of Business Venturing, vol. 3, p. 197-210.

BÉCHARD, J.P. (1996), Comprendre le champ de l'entrepreneurship, Cahier de recherche $\mathrm{n}^{0}$ 96-01-01, Chaire d'entrepreneurship Maclean Hunter, École des HEC de Montréal.

BIRCH, D.L. (1983), The Contribution of Small Enterprise to Growth and Employment, Program on Neighborhood and Regional Change, Massachusetts Institute of Technology, Cambridge, Mass., Harvard University Press.

BIRLEY, S. (1989), « The role of networks in the entrepreneurial process », Journal of Business Venturing, vol. 1, p. 107-117.

BlawATT, K. (1995), «Defining the entrepreneurs: a conceptual model of entrepreneurship », Actes du $12^{e}$ colloque annuel du CCPME (Conseil canadien de la PME et de l'entrepreneuriat), Thunder Bay, Ontario, octobre, p. 13-37.

Bolton, J.E. (1971), «Small firms : report of the Committee of inquiry on small firms », Président J.E. Bolton DSC. Présenté au Parlement (novembre), UK, HMSO (Her Majesty's Stationery Office) Cmnd 4811.

BRoCKHAUS, R.H. Sr. (1982), «The psychology of the entrepreneur », dans C.A. Kent et al. (éds), Encyclopedia of Entrepreneurship, Englewood Cliffs, N.J., Prentice-Hall, p. 39-57.

BROEHL, W.G. Jr. (1978), The Village Entrepreneur: Change Agents in India's Rural Development, Cambridge, Mass., Harvard University Press.

BULL, I. et G.E. WILLARD (1993), « Towards a theory of entrepreneurship », Journal of Business Venturing, vol. 8, n⿳0 3, p. 183-196.

Bull, I., H. ThOMAS et G. WILlaRd (éds) [1995], Entrepreneurship. Perspectives on Theory Building, Oxford, Pergamon Press.

BuChanAN, J.M. et A. Di PIERRo (1980), « Cognition, choice and entrepreneurship », Southern Economic Journal, vol. 46, p. 693-701. 
Burdeau, G. (1979), Le libéralisme, Paris, Éditions du Seuil.

Burns, P. et J.C. DEWhuRst (éds) [1989], Small Business and Entrepreneurship, Londres, MacMillan.

BYGRAVE, W.D. (1989a), «The entrepreneurship paradigm (I): a philosophical look at its research methodologies », Entrepreneurship Theory \& Practice, automne, p. 7-26.

BYGRAVE, W.D. (1989b), « The entrepreneurship paradigm (II) : chaos and catastrophes among quantum jumps?», Entrepreneurship Theory and Practice, hiver, p. 7-30.

BYGRAVE, W.D. (1993), «Theory building in the entrepreneurship paradigm », Journal of Business Venturing, vol. 8, $\mathrm{n}^{0} 3$, p. 255-280.

CANTILlON, R. (1755), Essai sur la nature du commerce en général, Londres, Fetcher Gyler. Aussi, édité avec une traduction anglaise et autre matériel, par Henry Higgs, C.B., Londres, MacMillan (1931).

CASson, M. (1982), The Entrepreneur: An Economic Theory, Oxford, Martin Robertson.

CHELl, E. (1985), «The entrepreneurial personality: a few ghosts laid to rest?», International Small Business Journal, vol. 3, $\mathrm{n}^{\circ} 3$, p. 43-54.

Chicha, J. et P.A. JULIEN (1979), «Les stratégies des PME et leur adaptation au changement », Département d'administration et d'économique, Université du Québec à Trois-Rivières.

ClARK, J.B. (1899), «The distribution of wealth : a theory of wages », Interest and Profits, New York et Londres, MacMillan.

Cochran, T.C. (1968), «Entrepreneurship», dans D.L. Sills (éd.), International Encyclopedia of he Social Sciences, Londres et New York, MacMillan et The Free Press, vol. 5, p. 87-91.

Cole, A.H. (1959), Business in its Social Setting, Cambridge, Mass., Harvard University Press.

Collins, O.F., D.G. Moore et D.B. Unwalla (1964), «The enterprising man », $M S U$ Business Studies, Bureau of Business and Economic Research, Graduate School of Business Administration, Michigan State University, East Lansing, Michigan.

Collins, O.F. et D.G. MoORE (1970), The Organization Makers : A Behavioral Study of Independent Entrepreneurs », New York, Appleton-Century-Crofts (Meredith Corp.).

COSSETTE, P. (1994a), « Développement d'une méthode systématique d'aide à la mise au point de la vision stratégique chez le propriétaire-dirigeant de PME: une étude exploratoire », dans J.J. Obrecht, Les PME/PMI et leur contribution au développement régional et international, Actes de la $39^{e}$ Conférence mondiale de l'ICSB, Strasbourg, p. 73-82. 
Cossette, P. (éd.) [1994b], Cartes cognitives et organisations, Québec, Presses de l'Université Laval ; Paris, Éditions Eska.

Covin, J.-G. et D.P. SLEVIN 1991), «A conceptual model of entrepreneurship as firm behavior», Entrepreneurship Theory and Practice, vol. 16, n ${ }^{\circ} 1$, p. 7-25.

CUNNINGHAM, J.B. et J.C. LISCHERON (1991), « Defining entrepreneurship », Journal of Small Business Management, vol. 29, $\mathrm{n}^{\circ} 1$, p. 45-67.

DEAKINS, D. (1996), Entrepreneurship and Small Firms, New York, McGraw-Hill.

DÉRY, R. et J.M. TOULOUSE (1994), La structuration sociale du champ de l'entrepreneurship: le cas du Journal of Business Venturing, Cahier de recherche $\mathrm{n}^{0}$ 94-06-02, Chaire d'entrepreneurship Maclean Hunter, École des HEC, Montréal.

DURAND, D.E. (1975), «Effects of achievement motivation and skill training on the entrepreneurial behaviour of black businessmen », Organizational Behaviour and Human Performance, vol. 14, $\mathrm{n}^{\circ} 1$, p. 76-90.

DURAND, D. et D. SHEA (1974), «Entrepreneurial activity as a function of achievement motivation and reinforcement control », The Journal of Psychology, vol. 88, p. 57-63.

DU TOIT, D.F. (1980), «Confessions of a successful entrepreneur », Harvard Business Review, novembre-décembre, p. 44-48.

ELLIS, W.H. (1983), Canadian Entrepreneurs : Innovators or Manipulators, Communication présentée lors du $2^{\mathrm{e}}$ Congrès international organisé par l'International Council of Small Business, Halifax, Nouvelle-Écosse (Canada), du 26 au 29 juin. Aussi dans K.H. Vesper (1982), Frontiers of Entrepreneurship Research, Wellesley, Mass., Babson Center for Entrepreneurial Studies, p. 16-24.

ELY, R.T. et R.H. HeSs (1893), Outline of Economics, New York, MacMillan.

EYSENK, H.J. (1967), «Personally patterns in various groups of businessmen », Occupational Psychology, vol. 41, p. 249-250.

FILION, L.J. (1987), Entrepreneurship : bibliographie choisie et une revue de la documentation essentielle sur le sujet, Cahier de recherche ${ }^{0}$ 87-03, Groupe de recherche en économie et gestion des petites et moyennes entreprises (GREPME), Université du Québec à Trois-Rivières (UQTR).

FILION, L.J. (1988), The Strategy of Successful Entrepreneurs in Small Business : Vision, Relationships and Anticipatory Learning, Thèse de doctorat, University de Lancaster, Grande-Bretagne (UMI 8919064) [Tome 1, 695 p.; Tome 2, 665 p.].

FILION, L.J. (1989a), «The design of our entrepreneurial learning system : identify a vision and assess your relations system ». Actes de la troisième conférence canadienne sur les études en entrepreneuriat, Université de Calgary, 28-30 septembre, p. 77-90. 
FILION, L.J. (1989b), «Le développement d'une vision: un outil stratégique à maîtriser », Revue internationale de gestion, vol. 14, $\mathrm{n}^{\circ} 3$, septembre, p. 24-34.

FILION, L.J. (1990a), «Vision and relations : elements for an entrepreneurial metamodel », Actes de la dixième conférence annuelle de Babson sur la recherche entrepreneuriale, Babson College, MA., 4-6 avril, publié dans N.C. Churchill et W.C. Bygrave (éds) [1990]. Frontiers of Entrepreneurship Research 1990. Proceedings of the Tenth Annual Babson College Entrepreneurship Research Conference, Babson Park, MA, Center for Entrepreneurial Studies, Babson College, p. 57-71.

FILION, L.J. (1990b), Les entrepreneurs parlent, Montréal, Les Éditions de l'entrepreneur, 1990, 303 p.

FILION, L.J. (1991a), "Vision and relations : elements for an entrepreneurial metamodel », International Small Business Journal, vol. 9, n² 2, janvier, p. 26-40.

FILION, L.J. (1991b), «Vision et relations : clefs du succès de l'entrepreneur », Montréal, Les Éditions de l'entrepreneur, 272 p.

Filion, L.J. (1992), «Critique du livre», J. Stanworth et C. Gray (éds), [1991], Bolton Twenty Years On. The Small Firm in the 1990s, Londres, Paul Chapman, Revue internationale PME, vol. 5, no 3-4, p. 171-189.

FILION, L.J., (1993), «Entrepreneur, organisation et apprentissage : nécessité de s'aménager un espace de soi, partie 1: l'entrepreneur et l'apprentissage », Revue Organisation, vol. 2, n 2 , été, p. 59-69.

FILION, L.J., (1994), «Entrepreneur, organisation et apprentissage : nécessité de s'aménager un espace de soi, partie 2 : entrepreneur, organisation et entreprise », Revue Organisation, vol. 3, $\mathrm{n}^{0} 1$, hiver, p. 47-55.

FILION, L.J. (1996a), «Différences dans les systèmes de gestion des propriétairesdirigeants, entrepreneurs et opérateurs de PME», à paraître dans Revue Canadienne des Sciences de l'Administration, décembre.

Filion, L.J. (1996b), «A marketing system for micro-enterprises », Communication présentée aux UIC/ AMA Research Symposia on Marketing and Entrepreneurship, Stockhom, Suede, 14-15 juin 1996, vol. 2, p. 150-166.

FunuYama, F. (1992), The End of History and the Last Man, New York, The Free Press.

FukuYama, F. (1994), Trust: The Social Virtues and the Creation of Prosperity, New York, The Free Press.

GARTNER, W.B. (1985), «A conceptual framework of describing the phenomenon of new venture creation », Academy of Management Review, vol. 10, no 4, p. 696-706.

GARTNER, W.B. (1990), «What are we talking about when we talk about entrepreneurship ?», Journal of Business Venturing, vol. 5, $\mathrm{n}^{\circ}$ 1, p. 15-29. 
GARTNER, W.B. et J.W. CARLAND et al. (1988), «Who is an entrepreneur? is the wrong question », American Journal of Small Business, vol. 12, $\mathrm{n}^{0} 4$, p. 11-39.

GASSE, Y. (1978), Characteristics, Functions and Performance of Small Firms : Owner-managers in Two Industrial Environments (2 volumes), Thèse de doctorat, Northwestern University, Evanston Ill., University Microfilm International, $n^{0} 79-3265$.

GASSE, Y. (1982), «Elaboration on the psychology of the entrepreneur », dans C.A. Kent et al. (1982), Encyclopedia of Entrepreneurship, Englewood Cliffs, N.J., Prentice-Hall, p. 57-66.

GIBB, A. et J. RITCHIE (1981), «Influence on entrepreneurship : a study over time», dans Bolton Ten Years On, Actes de la conférence U.K. Small Business Research, tenue du 20 au 21 novembre, Polytechnic of Central London.

GlueCK, W.F. (1977), Management, Hinsdale, Ill., The Dryden Press.

GUNDER, F.A. (1969), « Sociology of development and underdevelopment of sociology », dans N. Howel et al. Catalyst. Référence dans C.A. Kent et al. (éds) [1982] Encyclopedia of Entrepreneurship, Englewood Cliffs, N.J., Prentice-Hall, p. 58.

HAYEK, F.A, VON (1937), «Economics and knowledge», dans Economica, New Series, 4 (13-16), p. 33-54. (Presidential Address to the London Economic Club, 10 novembre 1936.) Réimprimé dans F.A. Hayek (1959), Individualism and Economic Order, Londres, Routledge and Kegan Paul, p. 33-56.

HAYEK, F.A, VON (1959), "The use of knowledge in society », American Economic Review (1945), 35, p. 519-530. Aussi dans Individualism and Economic Order (1959), Londres, Routledge et Paul Kegan, Chicago, University of Chicago Press (1948) (1957). Voir aussi « The meaning of competition » (1949), dans Individualism and Economic Order (1959), p. 92-106.

HÉBERT, R.F. et A.N. LINK (1982), The Entrepreneur : Mainstream Views and Radical Critiques, New York, Praeger.

Higgins, B.H. (1959), Economic Development : Principles, Problems, and Policies, New York, Norton.

HOFER, C.W. et W.D. BYGRAVE (1992), «Researching entrepreneurship », Entrepreneurship Theory and Practice, vol. 16, $\mathrm{n}^{\circ} 3$, p. 91-100.

HORNADAY, J.A. (1982), «Research about living entrepreneurs », dans C.A. Kent et D.L. Sexton et al. (éds), Encyclopedia of Entrepreneurship, Englewood Cliffs, N.J., Prentice-Hall, p. 20-34.

HoSELITZ, B.F. (1952), «Entrepreneurship and economic growth », American Journal of Economic Sociology, p. 97-106.

Hoselitz, B.F. (éd.) [1968], The Role of Small Industry in the Process of Economic Growth, Japan by Miyohei Shinohara, India by Douglas Fisher. The Hague, Paris, Mouton. 
Hull, D.L., J.J. Bosley et G.G. UdeEl (1980), «Renewing the hunt for the heffalump : identifying potential entrepreneurs by personality characteristics », Journal of Small Business Management, vol. 18, $\mathrm{n}^{\circ} 1$, p. 11-18.

HUNDALL, P.S. (1971), «A study of entrepreneurial motivation : comparison of fact - and slow - progressing small scale industrial entrepreneurs in Punjab, India », Journal of Applied Psychology, vol. 55, no 4, p. 317-323,

IBRAHIM, A.B. (1994), «Strategy types and small firm's performance: an empirical investigation », Journal of Small Business Strategy, vol. $4, \mathrm{n}^{\circ} 1$.

INNIS, H.A. (1930), The Fur-Trade in Canada : An Introduction to Canadian Economic History, Toronto, Les Presses de l'Université de Toronto (voir aussi édition révisée de 1956).

INNIS, M.Q. (éd.) [1956], Essays in Canadian Economic History (Harold Adam Innis). Toronto, Les Presses de l'Université de Toronto.

JULIEN, P.A. (1989), « The entrepreneur and economic theory », International Small Business Journal, vol. 7, n 3, p. 29-38.

JULIEN, P.A. (1990), « Vers une typologie multicritère des PME », Revue Internationale $P M E$, vol. 3, nos 3-4, p. 411-425.

Julien, P.A. et M. Marchesnay (1987), La petite entreprise, Paris, Vuibert.

Julien, P.A. et M. MARChESNAY (1996), L'entrepreneuriat, Paris, Economica.

KENNEDY, P. (1991), Naissance et déclin des grandes puissances, Paris, Éditions Payot.

Kent, C.A., D.L. SEXTON et K.H. VeSPER (éds) [1982], Encyclopedia of Entrepreneurship, Englewood Cliffs, N.J., Prentice-Hall.

KETS DE VRIES, M.F.R. (1985), « The dark side of entrepreneurship », Harvard Business Review, novembre-décembre, p. 160-167.

KIHLSTROM, R.E.et J.J. LAFFONT (1979), «A general equilibrium entrepreneurial theory of firm formation based on risk aversion ", Journal of Political Economy, vol. $87, n^{0} 4$, p. $719-748$.

KIRCHHOFF, B.A. (1992), « Entrepreneurship's contribution to economics », Entrepreneurship Theory and Practice, vol. 16, $\mathrm{n}^{\circ} 2$, p. 93-112.

KIRCHHOFF, B.A. (1994), Entrepreneurship and Dynamic Capitalism, Westfort, Conn., Praeger.

KIRZner, I.M. (1976), The Economic Point of View : An Essay in the History of Economic Thought, deuxième édition, Kansas City, Sheed and Ward.

KIRZNER, I.M. (1983), Perception, Opportunity and Profit: Studies in the Theory of Entrepreneurship, $1^{\text {re }}$ édition (1979) Chicago, University of Chicago Press. 
KNIGHT, F.H. (1921), Risk, Uncertainty and Profit, New York, Houghton Mifflin ; Chicago, University of Chicago Press (1971).

KUNKEL, J.H. (1965), «Values and behavior in economic development », Economic Development and Cultural Change, vol. 13, n 3, p. 257-277.

LAUFER, J.C. (1974), « Comment on devient entrepreneur », Revue française de gestion, vol. 2 , p. 18-29.

LAFUENTE, A. et V. SALAS (1989), «Types of entrepreneurs and firms : the case of new Spanish firms », Strategic Management Journal, vol. 10, p. 17-30.

LEFF, N.H. (1978), «Industrial organization and entrepreneurship in the developing countries : the economic groups », Economic Development and Cultural Change, vol. $26, n^{0} 4$, p. 661-675.

LEFF, N.H. (1979), «Entrepreneurship and economic development : the problem revisited », Journal of Economic Literature, vol. 17, $\mathrm{n}^{\text {os }}$ 1-2, p. 46-64.

LEIBENSTEIN, H. (1968), « Entrepreneurship and development », American Economic Review, vol. $38, \mathrm{n}^{\circ} 2$, p. 72-83.

Leibenstein, H. (1978), General X-Efficiency Theory and Economic Development, Londres, Oxford University Press.

LEIBENSTEIN, H. (1979), «The general X-efficiency paradigm and the role of the entrepreneur », dans M.J. Rizzio (éd.), Time, Uncertainty and Disequilibrium, Lexington, Mass., D.C. Heath, p. 127-139.

LORRAIN, J. et L. DUSSAULT (1988a), «Relation between psychological characteristics, administrative behaviors and success of founder entrepreneurs at the start-up stage », dans B.A. Kirchhoff et W.A. Long et al. (éds), Frontier of Entrepreneurship Research, Babson, p. 150-164.

LORRAIN, J. et L. DUSSAULT (1988b), «Les entrepreneurs artisans et opportunistes : une comparaison de leurs comportements de gestion », Revue internationale $P M E$, vol. $1, \mathrm{n}^{\circ} 2$, p. 157-176.

Low, M.B. et I.C. MACMILlAN (1988), « Entrepreneurship : past research and future challenges », Journal of Management, vol. 14, n² 2, p. 139-161.

LYNN, R. (1969), « Personality characteristics of a group of entrepreneurs », Occupational Psychology, vol. 43, p. 151-152. Voir aussi H.J. Eysenk (1967), G. Jessup et H. Jessup (1971).

MCClelland, D.C. (1961), The Achieving Society, Princeton, New Jersey, Van Nostrand. (Voir aussi nouvelle introduction pour ce même ouvrage, New York, Irvington Publishers (1976.)

MCClelland, D.C. (1971), «Entrepreneurship and achievement motivation: approaches to the science of socio-economic development », dans P. Lengyel (éd.), Approaches to the Science of Socio-Economic Development-Approches de la science et du développement socio-économique, Paris, UNESCO. 
MCGuIRE, J. (1964), Theories of Business Behavior, Englewood Cliffs, New Jersey, Prentice-Hall.

MCGUIRE, J.W. (1976), «The small enterprise in economics and organization theory », Journal of Contemporary Business, vol. 5, $\mathrm{n}^{0} 2$, p. 115-138.

MARX, K. (1844), Économie et philosophie, dans K. Marx, Euvres Économie, tome II, Paris, Gallimard, 1968, p. 1-141.

MARX, K. (1848), Le manifeste communiste, dans K. Marx, Euvres Économie, tome I, Paris, Gallimard, 1965, p. 157-195.

Meredith, G.G., R.E. NELSON, et P.A. NECK (1982), The Practice of Entrepreneurship, Genève, Bureau international du travail.

Miles, R.E. et C.S. SNOw (1978), Organizational Strategy, Structure, and Process, New York, McGraw-Hill.

MILl, J.S. (1848), Principles of Political Economy with Some of Their Applications to Social Philosophy, en deux volumes, neuvième édition, Londres, Longmann, Green (1886).

Miller, G.A. (1962), Psychology: The Science of Mental Life, Harmondsworth, Middlesex, England, Penguin Books (1982).

MINER, J. B., N.R. SMITH et J.S. BRACKER (1989), «Role of entrepreneurial task motivation in the growth of technologically innovative firms », Journal of Applied Psychology, vol. 74, $\mathrm{n}^{0} 4$, p. 554-560.

MineR, J. B. (1990), «Entrepreneurs, high growth entrepreneurs and managers : contrasting and overlapping motivational patterns », Journal of Business Venturing, vol. 5, p. 221-234.

Mulholland, R. (1994), «Approaches to entrepreneurship research », dans J. Mount (éd.), Actes du $11^{e}$ colloque annuel du CCPME - Conseil canadien de la PME et de l'entrepreneuriat, Winnipeg, 27-29 octobre, p. 122-133.

NECK, P. (1971), « Report on achievement motivation training program conducted in Uganda », dans C.A. Kent, D.L. Secton et al. (éds) [1982], Encyclopedia of Entrepreneurship, Englewood Cliffs, New Jersey, Prentice-Hall, p. 42.

Newman, P.C. (1981), The Acquisitors, Toronto, McClelland and Stewart.

OXENFELDT, A.R. (1943), New Firms and Free Enterprise: Pre-war and Post-war Aspects, Washington, D.C., American Council on Public Affairs.

PATEl, V.G. (1975), «Venture Assistance Experiments in India », Actes du Symposium international sur l'entrepreneurship et le développement de la nouvelle entreprise, été. Référence dans Kent et al. (éds) [1982], Encyclopedia of Entrepreneurship, Englewood Cliffs, New Jersey, Prentice-Hall, p. 42.

PEnRose, E.T. (1959), The Theory of the Growth of the Firm, Oxford, Basil Blackwell, New York, Wiley. 
Peterson, R. (1977), Petites et moyennes entreprises pour une économie équilibrée, Montréal, Le cercle du livre de France. Titre original : Small Businesses : Building a Balanced Economy (1977), Erin, Ontario, Press Porcepic.

PETERSON, R. et K. AINSLIE (éds) [1988], Understanding Entrepreneurship, Dubuque, Iowa, Kendall / Hunt.

PINCHOT, G. (1986), Intraprendre, Paris, Inter Édition. Titre original : Intrapreneuring, (1985), New York, Harper D. Row.

REYNOLD, P. (1991), «Sociology and entrepreneurship : concepts and contributions », Entrepreneurship Theory and Practice, vol. 16, $\mathrm{n}^{\circ} 2$, p. 47-70.

RosA, P. et A. Bowes (1990), Entrepreneurship: Some Lessons of Social Anthropology, E.C.S.B. $4^{\text {th }}$ Workshop on Research in Entrepreneurship, Université de Cologne.

Rosenberg, N. et L.E. Birdzell Jr. (1986), How the West Grew Rich, New York, Basic Books.

ROTTER, J.B. (1966), Generalised Expectations for Internal Versus External Control of Reinforcement, American Psychological Association (Psychological Monographic, généralités et appl. 80, $n^{0} 1$, entièrement $n^{0} 609$ ).

SAY, J.B. (1803), Traité d'économie politique : ou, simple exposition de la manière dont se forment, se distribuent et se consomment les richesses. Traduction de Treatise on Political Economy: On the Production, Distribution and Consumption of Wealth, New York, Kelley, 1964 (1 $1^{\text {re }}$ édition, 1827).

SAY, J.B. (1815), De l'Angleterre et des Anglais, Paris, Arthur Bertrand.

SAY, J.B. (1816), England and the English People, $2^{\mathrm{e}}$ édition (1816), par John Richter, Londres, Sherwood, Neely et Jones, Traduction du livre précédent (1815).

SAY, J.B. (1839), Petit volume contenant quelques aperçus des hommes et de la société, $3^{\mathrm{e}}$ édition entièrement refondue sur les manuscrits laissés par l'auteur et publiée par Horace Say, son fils, Paris, Chez Guillaumin, Libraire.

SCASE, R.et R. GoFfEE (1980), The Real World of the Small Business Owner, Londres, Croom Helm.

SCHLOSS, H.H. (1968), « The concept of entrepreneurship in economic development », Journal of Economic Issues, juin, p. 228-232.

SChollhammer, H. (1980), Analysis and Assessment of Internal Corporate Entrepreneurship Strategies, Los Angeles, Californie, Graduate School of Management, UCLA.

SCHRAGE, H. (1965), « The R\&D entrepreneur : profile of success », Harvard Business Review, novembre-décembre, p. 56-69.

SCHUMPETER, J.A. (1928), «Der Unternehmer», dans Elster Ludwig et al. (éds) [1928], Handwörterbuch der Staatswissenschaften (4e édition, Jena $1928: 483$ ). Référence dans H. Hartmann (1959), «Managers and entrepreneurs : a useful distinction », Administrative Science Quarterly, vol. 3, n 3, p. 429-451. 
SCHUMPETER, J.A. (1934), The Theory of Economic Development, publié en allemand (1912), première édition en anglais, Cambridge, Mass., Harvard University Press, 1934, aussi Cambridge, Mass., Harvard Economic Studies, vol. 46, Londres, Oxford University Press.

SCHUMPETER, J.A. (1954), History of Economic Analysis, édité par Elizabeth Boody Schumpeter, New York, Oxford University Press, aussi Londres, George Allen et Unwin (6 édition, 1967).

SEXTON, D.L.et J.D. KASARDA (éds) [1992], The State of the Art of Entrepreneurship, Boston, PWS-Kent Publishing.

SEXTon, D.L.et R.W. SMILOR (éds) [1986], The Art and Science of Entrepreneurship, Cambridge, Mass., Ballinger.

SINGH, N.P. (1970), "N/ Ach, risk-taking and anxiety as related to age, years of schooling, job-experience and family », Psychologia, vol. 13, p. 113-116.

SINGH, N.P.et K. SINGH (1972), «Risk-taking among agricultural and business entrepreneurs of Delhi », Psychologia, vol. 15, p. 175-180.

SMIth, A. (1776), An Enquiry Into the Nature and Cause of the Wealth of Nations, Londres, Edwin Cannan. Aussi, Londres, Methuen (1950), Irwin (1963). Réédition en deux volumes. Voir en particulier l'édition par James E. Thorold Rogers, Oxford, Clarendon Press (1869).

SMITH, N.R. (1967), The Entrepreneur and His Firm: The Relationship between Type of Man and Type of Company, Bureau of Business Research, East Lansing, Michigan, Michigan State University Press.

SMITH, N.R. et J.B. MINER (1983), «Type of entrepreneur, type of firm, and managerial motivation: implications for organizational life cycle theory », Strategic Management Journal, vol. 4, p. 325-340.

SOMBART, W. (1928), Le bourgeois - Contribution à l'histoire morale et intellectuelle de l'homme économique moderne, Paris, Petite bibliothèque Payot.

StANWORTH, J. et J. CURRAN (1973), Management Motivation in the Smaller Business, Epping, Essex, Angleterre, A Gower Press Special Study.

Stanworth, J., A. Westrip, D. WATkins et J. Lewis (1982), Perspectives on a Decade of Small Business Research: Bolton Ten Years On, Aldershot, Hampshire, Angleterre, Gower.

Stanworth, J. et C. Gray (éds) [1991], Bolton, 20 Years on. The Small Firm in the 1990 's, Londres, Chapman.

STEVENSON, H.H. et C. JARILlo (1990), «A paradigm of entrepreneurship: entrepreneurial management », Strategic Management Journal, vol. 11, été, p. 17-27.

Storey, D.J. (1982), Entrepreneurship and the New Firm, Londres et Canberra, Croom Helm. 
STOREY, D.J. (1994), Understanding the Small Business Sector, Londres et New York, Routledge.

TAWNEY, R.H. (1947), Religion and the Rise of Capitalism, New York, Penguin.

Timmons, J.A. (1971), «Black is beautiful - Is it bountiful ? », Harvard Business Review, novembre-décembre, p. 81-94.

Timmons, J.A. (1973), « Motivating economic achievement : a five-year appraisal», Actes American Institute of Decision Sciences, novembre, Boston. Référence dans J.A. Timmons (1978a), «Characteristics and role demands of entrepreneurship », American Journal of Small Business, vol. 3, n 1, p. 5-17.

Timmons, J.A. (1978), «Characteristics and role demands of entrepreneurship », American Journal of Small Business, vol. 3, $\mathrm{n}^{0} 1$, p. 5-17.

Toulouse, J.M. (1979), L'entrepreneurship au Québec, Montréal, Les Presses HEC et Fides.

Toulouse, J.M. et G.A. BRENNER (1992), Activités d'affaires et groupes ethniques à Montréal, Cahier de recherche 92-09-02, Chaire d'entrepreneurship Maclean Hunter, École des HEC, Montréal.

ToYnbee, A. (1994), La grande aventure de l'humanité, Paris, Payot.

VACHET, A.C. (1988), L'idéologie libérale. L'individu et sa propriété, Ottawa, Presses de l'Université d'Ottawa.

VÉRIN, H. (1982), Entrepreneurs, entreprises, histoire d'une idée, Paris, Presses universitaires de France.

VeSPER, K.H. (1980), New Venture Strategies, Englewood Cliffs, N.J., Prentice-Hall.

VESPER, K.H. (éd.) [1985], Entrepreneurship Education 1985, Wellesley, Mass., Babson College, Center for Entrepreneurial Studies.

VESPER, K.H. (éd.) [1993], Entrepreneurship Education 1993, Los Angeles, CA, The Anderson School, University of California.

Weber, M. (1930), The Protestant Ethic and the Spirit of Capitalism, traduit par Talcott Parsons, Londres, Allen and Unwin.

WELSCH, H.P. (1992), International Entrepreneurship and Small Business Bibliography, Chicago, De Paul University.

Woo, C.Y., A.C. Cooper et W.C. Dunkelberg (1991), "The development and interpretation of entrepreneurial typologies », Journal of Business Venturing, vol. $6, \mathrm{n}^{0} 2$, p. 93-114.

WORTMAN, M. et W. BIRKENHOLZ (1991), «Entrepreneurship research on a global basis : an empirically based model », 36th Annuel World Conference ICSB, Vienne, Proceedings, vol. 1, p. 67-77. 